\title{
Can Oppositely Charged Polyelectrolyte Stars Form a Gel? A Simulational Study
}

\author{
Andrea Tagliabue ${ }^{\dagger}$ Jonas Landsgesell, $^{\ddagger}$ Massimo Mella,${ }^{\dagger}$ and Christian Holm ${ }^{*}, \ddagger$ \\ $\dagger$ Dipartimento di Scienza ed Alta Tecnologia, Università degli Studi dell'Insubria, via \\ Valleggio 9, 22100, Como, Italy \\ $\ddagger$ Institute for Computational Physics, University of Stuttgart, Allmandring 3, Stuttgart, \\ 70569, Germany \\ E-mail: holm@icp.uni-stuttgart.de
}

\begin{abstract}
We present a Langevin molecular dynamics study of an equimolar mixture of monodispersed oppositely charged di-block four-armed polyelectrolyte stars. We use an implicit solvent coarse-grained representation of the polyelectrolyte stars and varied the length of the terminal charged blocks that reside on each arm. By varying the polymer concentration we computed P-V diagrams and determined the free-swelling equilibrium concentration with respect to a pure water reservoir as a function of the charged block length. We investigate various structural properties of the resulting equilibrium structures, like the number of ionic bonds, dangling arms, isolated stars, and cluster sizes. The ionic bonds feature a broad distribution of the number of arms involved and also display a distribution of net charges peaked around the neutral ionic bond. Our main result is that for charged block length equal to 4 and 5 ionized beads the resulting macro-aggregate spans the box and forms a network phase. Furthermore, we investigated the dynamics of ionic bonds, and computed their lifetimes and restruc-
\end{abstract}


turing dynamics. The bonds are weak enough to allow a network restructuring under thermal fluctuations but are still strong enough to yield a stable gel phase.

September 6, 2020

\section{Introduction}

A polyelectrolyte is a polymer composed of monomers containing dissociable groups which release counterions $\left(\right.$ e.g. $\left.\mathrm{Na}^{+}\right)$upon dissolution in solvents, e.g. water. If the base polymer is a star polymer, it is called correspondingly a polyelectrolyte star. If polyelectrolytes get chemically cross-linked they can form polyelectrolyte gels, and since they are water soluble one sometimes also calls them hydrogels. These gels possess a huge swelling capacity in aqueous solution can absorb water in amounts of up to a few hundred times their dry mass. This makes them ideal base materials for super-absorbers in hygiene products ${ }^{1}$, biomedical ${ }^{2-9}$ and agricultural ${ }^{10-12}$ applications, and even for desalination purposes ${ }^{13-16}$. In contrast to bulk materials, polyelectrolyte micro- and nano-gels are being investigated as nano-reactors ${ }^{17-19}$ or as carriers for controlled drug release ${ }^{20-24}$.

Chemical cross-linking is not the only way to form a gel. There are so called physical gels that form via reversible bonds that can be based on various physical non-covalent interactions such as hydrogen bonds, $\pi-\pi$-stacking, hydrophobic forces, or van der Waals or ionic interactions. The connectivity of the gel constituents is therefore partially or not at all fixed. This leads to the fact that physical gels are normally less structured ${ }^{25-28}$ than chemical gels which are often formed with tetra-functional nodes.

Physically cross-linked gels can have certain advantages; for example, a bond rupture event is reversible, and such gels can be to a certain degree self-healing. Of special interest could be ionic bonds, since they are tunable via many parameters. As an example, they can be formed and destroyed dynamically, and also their strength can be tuned by addition of salt, changes in the relative dielectric constant of the solution via adding co-solvents, or 
varying the solution $\mathrm{pH}$ if the dissociable groups are weak. For example, one could tune cargo encapsulation and a following release on changing some of the stimuli, e.g. the ionic strength.

The investigation of physically cross-linked networks by simulations are scarce. Exceptions worth mentioning are the investigations of associating polymers, so-called telechelic polymer chains ${ }^{29-34}$, as well as the investigation of ionomers ${ }^{35-39}$ and neutral block starshaped copolymers ${ }^{34}$; these, however, do not fall into the class of strongly swelling, ionically reversible cross-linked stars. Many theories also deal with the swelling of chemical gels, but much less with the swelling behaviour of physical ones. As a notable exception we mention the works of Tanaka and others ${ }^{40-46}$ on the properties of physically cross-linked ionic gels. Since a popular way of synthesizing chemically cross-linked gels with a low polydispersity is based on tetra-PEG ansatz from Sakai and co-workers ${ }^{47}$, in this work we will investigate the physical gelation properties of four-armed polyelectrolyte stars, where one star species carries positively charged blocks, and the other star species carries negatively charged blocks. This could, in principle, lead to a regular tetra-functional network with matching charged blocks if the system is perfectly monodispersed. To our knowledge there have been no previous simulations performed with ionically bonded star polyelectrolytes. Investigations of regular charged polyelectrolyte copolymer networks using a thermodynamic model has been done by the group of Patrickios in a series of publications ${ }^{48-50}$. Their model predicts that such a network has a discontinuous transition from a homogeneous to a micellar phase. The micellar-ordered phases are similar to those known from di-block copolymer melts. We therefore present the first exploratory simulation to study the gelation properties of an equimolar solution mixture of oppositely charged star polyelectrolytes. For the sake of simplicity we avoid to add any salt ion or counterion in solution, and we treat the system as perfectly mono-dispersed.

The article is structured as follows: in Section 2 we will present our model and the used simulation methods, followed by our results in Section 3. We will conclude with a summary 
of our main results and an outlook for further studies in Section 4 .

\section{Methods and Model}

\subsection{The model}

Our system consists of a cubic simulation box of length $L$, with periodic boundary conditions in all the three dimensions, which contains $N_{\mathrm{s}}=64$ star polymers. The latter are treated as a coarse-grained "bead-spring" model and consist of $N_{\mathrm{a}}=4$ arms tethered to a common central monomer, or "nucleus". Each arm is composed of $N_{\text {mono }}^{(\text {a) }}=10$ monomers, so that the number of beads in each star-like polymer is $N_{\text {mono }}^{(\mathrm{s})}=N_{\text {mono }}^{(\mathrm{a})} N_{\mathrm{a}}+1=41$, and the total number of monomers in the cell is $N_{\text {mono }}^{(\text {tot })}=41 N_{\mathrm{s}}=2624$. In the following, parameter and properties that refer to monomers, individual arms and stars are labelled with "mono", "a", and "s" as subscripts, respectively. Furthermore, during the discussion we will use italic capital letters $(A, B$, etc. $)$ as star indexes, italic lowercase letters $(i, j$, etc. $)$ as monomer indexes, and italic Greek lowercase ones ( $\alpha, \beta$, etc.) as arms indexes.

Each arm is structured as a AB-block copolymer, where "A" is the terminal part of the chain and is composed of $\Omega$ beads carrying each one quenched monovalent charge (i.e., they behave as strong electrolytes), whereas the part " $\mathrm{B}$ " is directly connected to the nucleus

and is composed by $N_{\text {mono }}^{(\mathrm{a})}-\Omega$ neutral beads; the nucleus is neutral itself. We provide a pictorial description of our block copolymer model in Figure 1. Half of the stars in solution $\left(N_{\mathrm{s}}^{+}\right)$carry positive charges, while the remaining half $\left(N_{\mathrm{s}}^{-}\right)$is negatively charged, so that $N_{\mathrm{s}}^{+}=N_{\mathrm{s}}^{-}=N_{\mathrm{s}} / 2=32$, and the system is overall electroneutral.

All monomers interact via a Weeks-Chandler-Anderson potential to simulate their excluded volume ${ }^{51}$ :

$$
U_{\mathrm{LJ}}\left(r_{i j}\right)= \begin{cases}4 \epsilon\left[\left(\frac{\sigma}{r_{i j}}\right)^{12}-\left(\frac{\sigma}{r_{i j}}\right)^{6}+\frac{1}{4}\right] & \text { if } r_{i j}<r_{\mathrm{cut}} \\ 0 & \text { if } r_{i j} \geq r_{\mathrm{cut}}\end{cases}
$$


here, $r_{i j}$ is the distance between two interacting monomer $i$ and $j, \epsilon=k_{B} T$ is the depth of the potential, $\sigma$ is the range of interaction, and $r_{\text {cut }}=2^{\frac{1}{6}} \sigma$ is the cutoff radius. Bonds between adjacent beads are simulated via finitely extensible non-linear elastic (FENE) potentials, ${ }^{52}$

$$
U_{\mathrm{FENE}}\left(r_{i j}\right)=-\frac{1}{2} k r_{\max }^{2} \ln \left(1-\left(\frac{r_{i j}}{r_{\max }}\right)^{2}\right)
$$

where $k=30 \epsilon / \sigma^{2}$ is the spring constant and $r_{\max }=3 \sigma$ is the maximum allowed elongation. Arms are connected to the central bead via the same FENE potential. No angular terms have been added to the total potential, so that the polymer chains are fully flexible, and tethered arms can easily rearrange around the nuclei. Electrostatic interactions are calculated by the $\mathrm{P}^{3} \mathrm{M}$ method ${ }^{53,54}$, with errors ${ }^{55}$ set to $10^{-3}$. The solvent is treated as a uniform dielectric. The Bjerrum length has the value $\lambda_{B}=e^{2} / 4 \pi \varepsilon k_{B} T=2 \sigma$, where $e$ is the elementary charge and $\varepsilon$ is the permittivity of the medium. Setting $\sigma=3.55 \AA$ results in the typical Bjerrum length of the water at room temperature, $\lambda_{B}=7.10 \AA$.
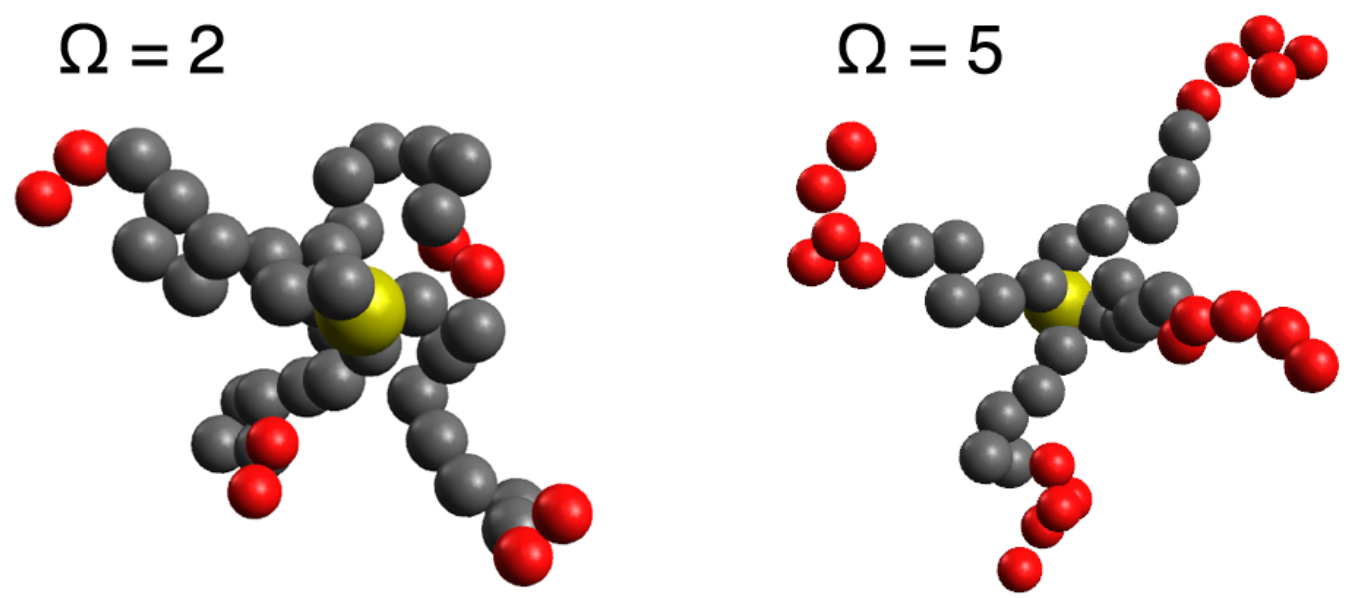

Figure 1: Models of tetra-functional strong polyelectrolyte stars simulated in this work. The snapshots were taken from simulations at very high dilution. $\Omega$ is the number of charged monomer at the end of each arm. Colour scheme: neutral monomers in grey, (positively) charged monomers in red, central beads in yellow. 


\subsection{Simulation methods}

Molecular dynamics simulations are performed in the NVT ensemble using a Langevin ther$\operatorname{mostat}^{56}$ according to

$$
m_{i} \ddot{\mathbf{r}}_{i}=-\gamma \dot{\mathbf{r}}_{i}+\mathbf{F}_{i}+\mathbf{R}_{i}
$$

where $m_{i}=1$ is the mass of the $i$-th monomer, $\dot{\mathbf{r}}_{i}, \ddot{\mathbf{r}}_{i}, \mathbf{F}_{i}$ and $\mathbf{R}_{i}$ are, respectively, its velocity, its acceleration, and the conservative and the random forces acting on it, and $\gamma=\sigma^{-1} /(m \epsilon)^{1 / 2}$ is the friction coefficient. Random forces act on each monomer independently and obey the fluctuation-dissipation theorem, i.e. $\left\langle R_{i k}\right\rangle=0$ and $\left\langle R_{i k}(t) R_{j l}\left(t^{\prime}\right)\right\rangle=$ $2 \gamma k_{B} T \delta_{i j} \delta_{k l} \delta\left(t-t^{\prime}\right)$. Equation 3 is integrated by a velocity Verlet algorithm with a time

step $\delta t=0.01 \sigma(m / \epsilon)^{1 / 2}$; thus our system time unit $\tau=\sigma \sqrt{m / \epsilon}$ contains 100 integration steps. All simulations have been performed with the software package ESPResSo ${ }^{57}$. In the following all length and time units are expressed in multiples of $\sigma$ and $\tau$, respectively, unless otherwise noted.

The equilibrium between the system and pure water is called "free-swelling equilibrium". In the canonical ensemble it is obtained at the minimum of the Helmholtz free energy $F$ as a function of the volume $V$, that is $\partial F / \partial V=-P=0$, where $P$ is the volume averaged virial pressure, so that the equilibrium volume $V_{\text {eq }}=L_{\text {eq }}^{3}$ (and, consequently, the equilibrium concentration of the species $\left.C_{\mathrm{s}, \mathrm{eq}}\right)$ is defined at $V$ where the pressure $P$ is equal to 0 .

In order to identify $L_{\mathrm{eq}}$ as a function of the number of terminal charges, we performed a series of simulations varying the box length $L$ for species with $\Omega=1,2,3,4$, and 5 . For each system type, three different simulation protocols have been implemented; these are:

single-points protocol: for each value of the desired box length, $L \in\left[L_{\min }, L_{\max }\right]$, we simulate the system starting from a random solution of $N_{\mathrm{s}}$ stars. The system is initially thermalized for a time $t_{\text {therm, }}$, then we take a time-average measure of all desired properties during a simulation time $t_{\text {sim }}$.

expansion protocol: we start simulating a random solution of $N_{\mathrm{s}}$ stars at a box length 
$L=L_{\min }$ and ensure ourselves that we have a positive volume average virial pressure $P$ (e.g., vide infra Figure 2). The system is thermalized for a time $t_{\text {therm }}$, then properties are collected for a time $t_{\mathrm{sim}}$. Once the simulation at $L=L_{\mathrm{min}}$ is done, we increase the box length $L$ by a quantity $\Delta L$ (in order to obtain an isotropic expansion in $V$ ), we thermalize the last configuration obtained at the previous volume for a time $t_{\text {therm }}$, and then we collect properties for a time $t_{\mathrm{sim}}$. The described process is then repeated until the system reach a desired box length $L=L_{\max }$.

compression protocol: we use the same scheme described for the "expansion" simulations, but starting from a box length $L=L_{\max }$ and decreasing it by a quantity $\Delta L$ at each step until it reaches $L_{\min }$.

For all the cases, we set $L_{\min }=16 \sigma, L_{\max }=50 \sigma, t_{\text {therm }}=10^{5} \delta t$ and $t_{\text {sim }}=10^{6} \delta t$. We also chose $\Delta L=2 \sigma$ except for the ranges of box length values in which the systems are expected to be near the free-swelling equilibrium; in such ranges we increased the sampling resolution up to $\Delta L=0.25 \sigma$. For each simulated value of $L, 50$ independent simulations were performed in order to improve the sampling of possible configurations that may be hindered by high energy barriers. Our data represents averaged results accompanied by their standard errors. Subsequently, $L_{\text {eq }}$ has been calculated by fitting the $L$ values via a second-order polynomial, and we performed 50 independent simulations at $L_{\text {eq }}\left(t_{\text {therm }}=10^{5} \delta t, t_{\text {sim }}=10^{6} \delta t\right)$ in order to collect information about structural and dynamical properties of aggregates in solution.

\section{Results and discussion}

\subsection{Finding the free-swelling equilibrium}

Figure 2 shows the behaviour of the volume averaged virial pressure $P$ of the system as a function of the box length $L$ for species with a different number of terminal charged monomers, $\Omega=1,2,3,4$, and 5 . Results are obtained implementing the "single-points" 


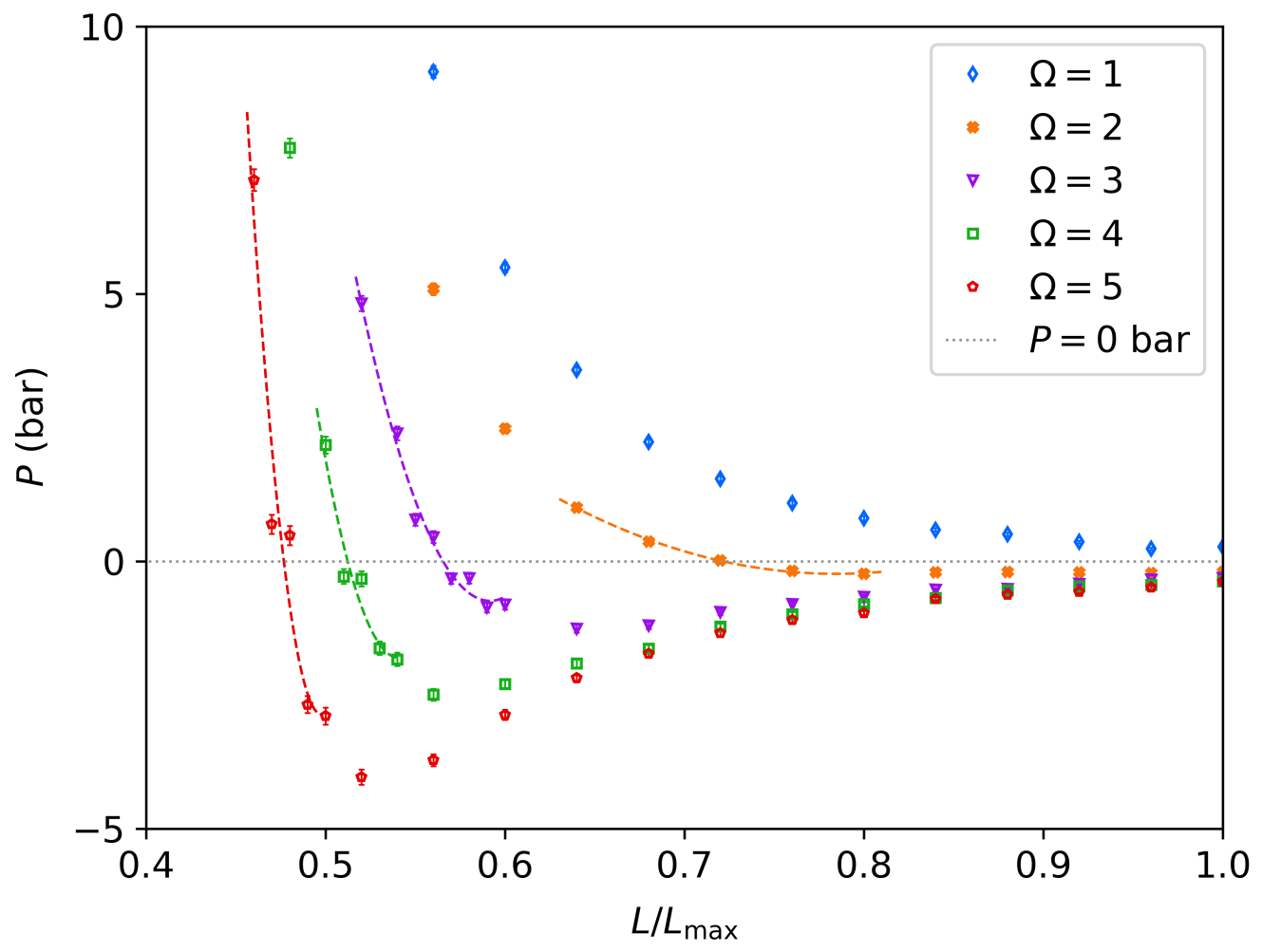

Figure 2: The isotropic pressure $P$ (bar) vs $L / L_{\max }$ for different values of $\Omega$ obtained via the "single points" simulation protocol. The dashed lines are the quadratic fits performed in order to identify the $L_{\text {eq }}$ values (the fitting parabolic function is used as the simplest option available and it has no physical significance). The dotted grey line is only a guide to the eye for discerning positive and negative pressure values. Standard error bars are included everywhere, but are sometimes smaller than the plot symbols. 
protocol described in Section 2.2. For $\Omega=1$ (i.e., star polymers carrying only a monovalent terminal bead on each arm), we observe a positive pressure over all $L$ ranges simulated. At high $L / L_{\max }$ values, i.e. at high concentration (see also Figure S2Volume averaged virial pressure $P$ (bar) as a function of species concentration $C_{\mathrm{S}}(\mathrm{mol} / \mathrm{L})$ for the four $\Omega$ values. The dotted grey line is only a guide for the eye for discern positive and negative pressure values. Standard error bars are smaller than plot symbols where they're not visible.figure.caption.7 in the Electronic Supporting Information (ESI)) $P$ starts to strongly increase due to the monomer excluded volume interactions, which oppose the compression preventing particles overlaps. As the box volume increases, the system becomes more diluted, and $P$ tends to $0^{+}$

For $\Omega \geq 2$ we observe a different behaviour. As the number of terminal charges increases, $P$ decreases for all simulated box lengths. We attribute this to the progressively stronger electrostatic attraction between the two oppositely charged species. At small box length values we observe, as for the $\Omega=1$ case, positive values of $P$ arising from the internal pressure generated by monomer excluded volumes. Unlike for the $\Omega=1$ case, however, increasing the box volume results in non-monotonic $P$ curves, and we find regions of negative pressure. As the system becomes more diluted, the isotropic pressure $P$ asymptotically converges to 0 from below. We always find one box length $L_{\text {eq }}$ for which $P\left(L_{\text {eq }}\right)=0$ and that could be a candidate system to possess an equilibrium phase against a pure water system. In order to precisely determine $L_{\text {eq }}$, for each $\Omega$ value $(\Omega \geq 2)$ we fitted the points near $P=0$ with a parabolic function, weighting each data point with its respective statistical error (see the dashed lines in Figure 2). In this way we found $L_{\mathrm{eq}} / \sigma=36.07,28.27,25.65$, and 23.84 for $\Omega$ values 2 to 5 , respectively. These correspond to species molar concentrations $C_{\mathrm{s} \text { eq }}$ equal to $2.55 \cdot 10^{-2}, 5.40 \cdot 10^{-2}, 7.05 \cdot 10^{-2}$ and $8.75 \cdot 10^{-2} \mathrm{~mol} / \mathrm{l}$, respectively. Figure 3 displays selected snapshots for the various systems at free-swelling equilibrium.

In order to check if our single-points simulation protocol is able to correctly sample the system at equilibrium, i.e. that we do not run into metastable configurations, we performed 

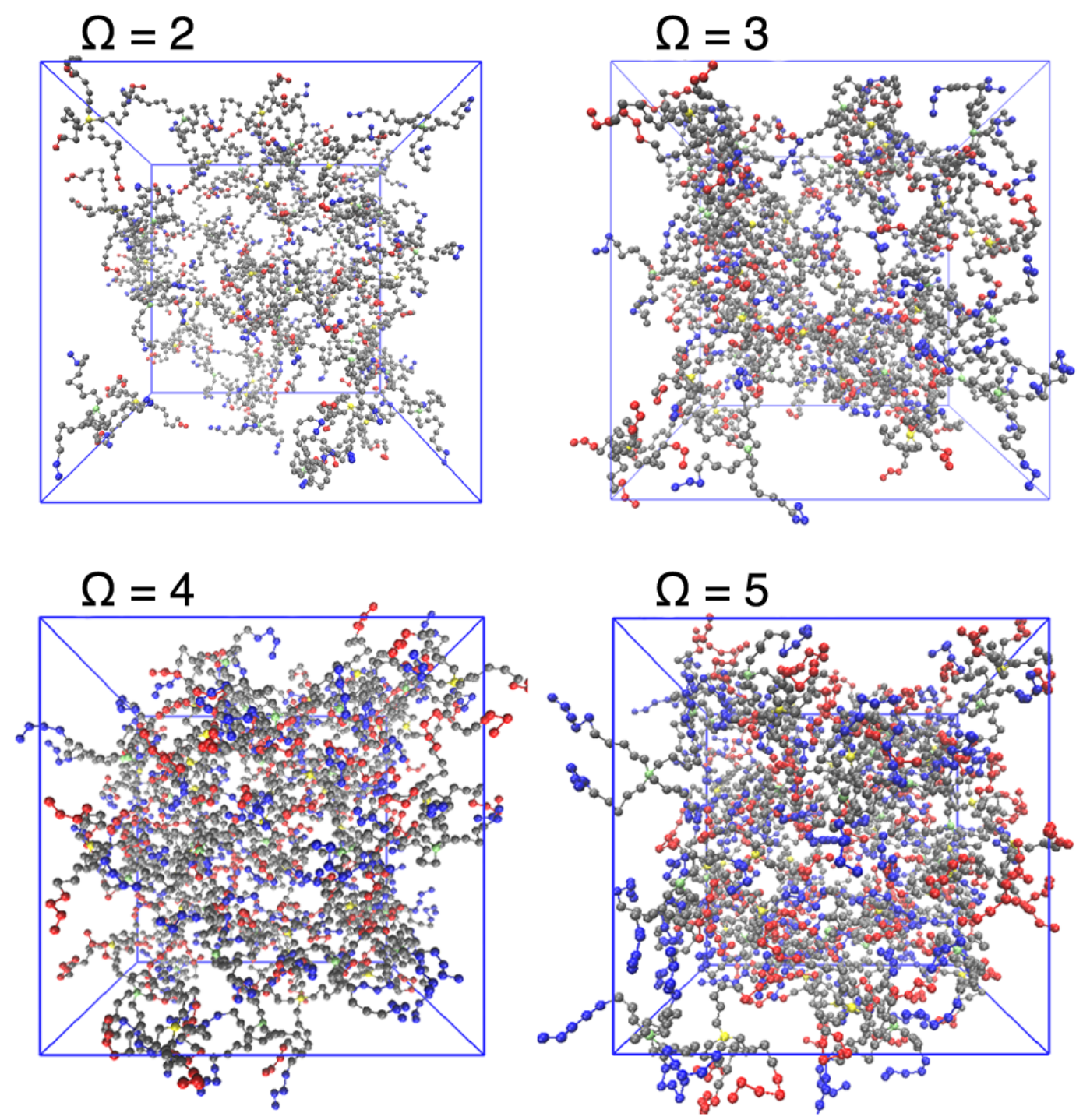

Figure 3: Snapshots for system with various $\Omega$ values at the free-swelling equilibrium. The diameter of all monomers has been reduced by roughly one half with respect to the real one in order to improve the clarity of the pictures. Colour scheme: neutral monomers in grey, positively charged monomers in red, negatively charged monomers in blue, the nuclei of positive stars in yellow, the nuclei of negative stars in lime. 


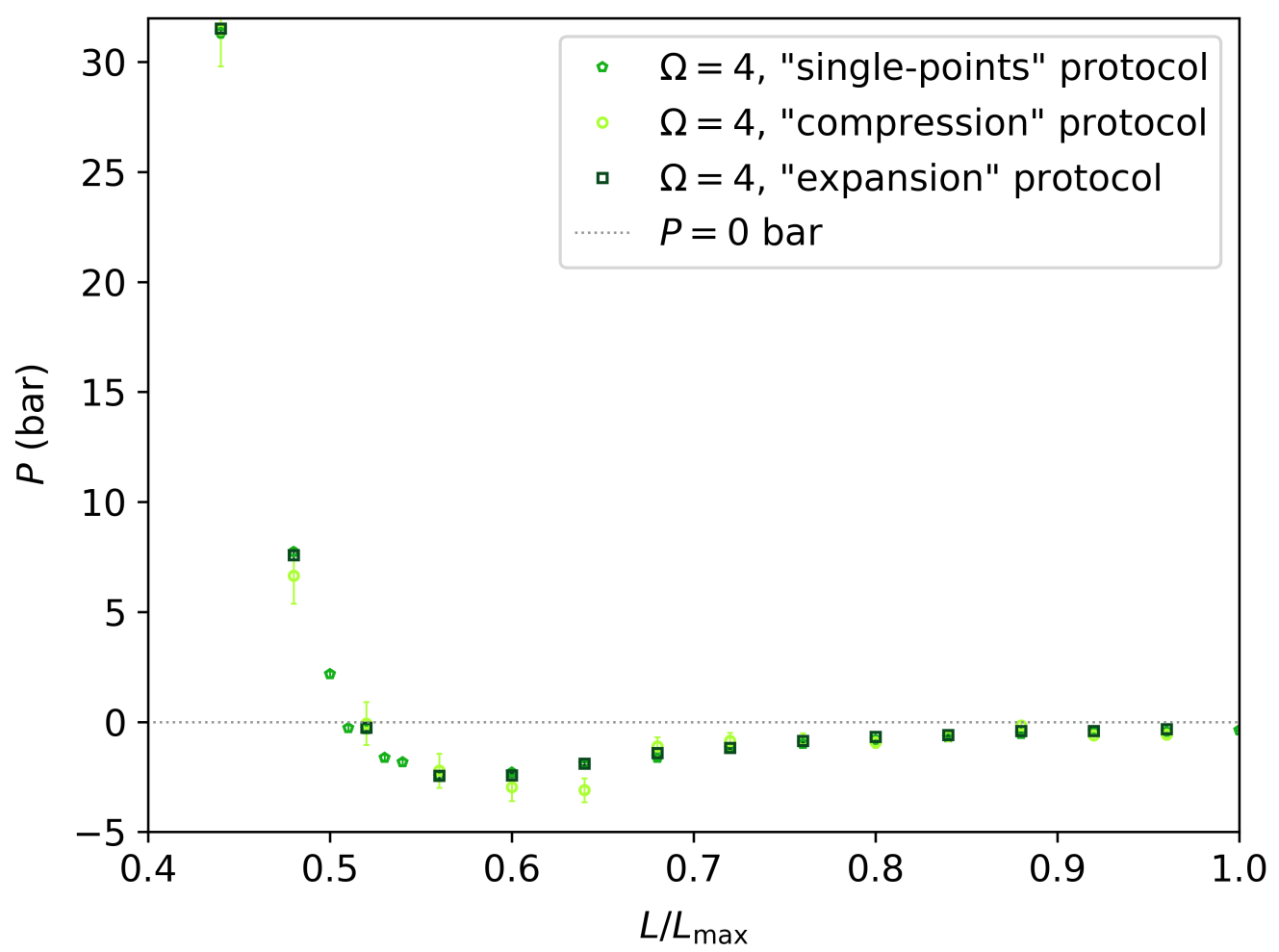

Figure 4: Comparison between the results obtained via the three different protocols described in Section 2.2 for the $\Omega=4$ case. The dotted grey line is a guide to the eye to discern positive and negative pressure values. Standard error bars are included everywhere, but are sometimes smaller than the plot symbols. 
the same simulations with the other two protocols described in section 2.2. Figure 4 displays the results for the three different schemes for $\Omega=4$ (the worst performing case), while the results for the $\Omega=3$ and 5 cases are reported in the ESI (Figure S4). From Figure 4, we infer that even for the worst case the results obtained via the three different protocols are in good agreement within error bars, hence we are confident that we are sampling the system in an ergodic way at each box length value.

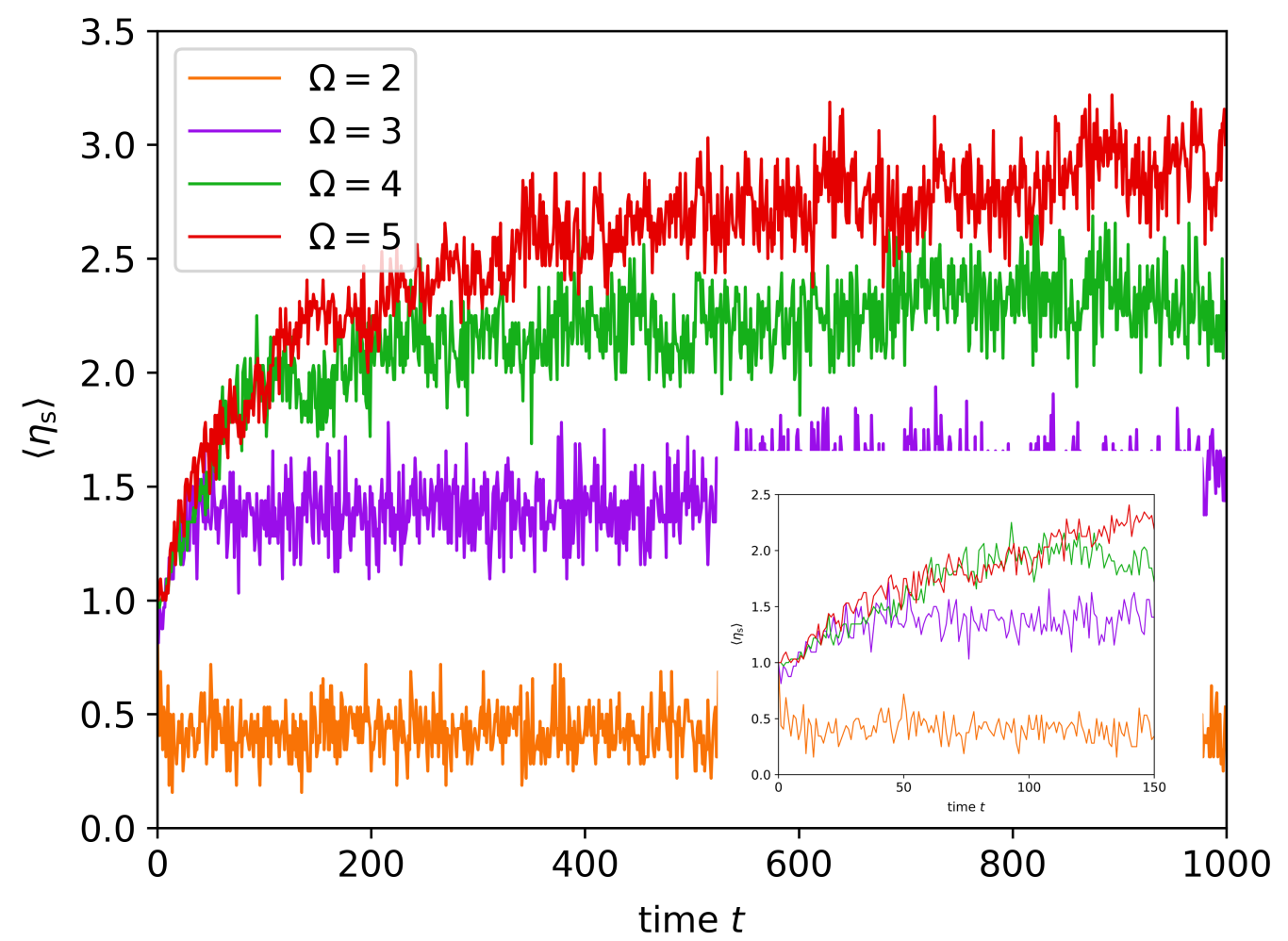

Figure 5: Average number of stars in contact with a given oppositely charged star $\eta_{\mathrm{s}}$ versus the simulation time $t$ for different $\Omega$ values. The inset shows the initial part of the simulation.

Since, for the sake of simplicity, we simulated only systems with an equimolar mixture of oppositely charged, but otherwise identical stars, the smallest stable supramolecular aggregates in solution are dimers composed by two oppositely charged stars. In order to investigate the stability of the latter with respect to the aggregates observed at $P=0$ (see Figure 3), we decided to monitor the evolution in time of a solution of 32 pre-assembled dimers.

To set up the system, for each $\Omega$ value we thermalized (for a time $t=10^{4}$ ) a single dimer 
at very low concentration. The dimers resulted stable for $\Omega \geq 2$, whereas for $\Omega=1$ the two polyelectrolytes frequently dissociate and only transient dimeric interactions were observed during the simulation. Thus, for $\Omega \geq 2$ species, 32 pre-equilibrated random configurations of such dimers have been homogeneously placed inside a simulation box of side length $L=42 \sigma$ $\left(L / L_{\max }=0.84\right)$, and the system was simulated for a time $t=10^{4}$.

To monitor the temporal evolution of the dimers, we define $\eta_{\mathrm{s}, A}$ as the number of oppositely charged stars in contact with a given star $A$ (vide infra Figure 6 ). Hence, in order to investigate the stability of the dimers with respect to clusters composed by more than 2 stars, we monitor the evolution of the value of $\eta_{\mathrm{s}, A}$ averaged over all the stars in solution, $\left\langle\eta_{\mathrm{s}}\right\rangle=\sum_{A}^{N_{\mathrm{s}}} \eta_{\mathrm{s}, A}$. We define a positive (negative) star $A$ to be "in contact" with a negative (positive) star $B$ if it is possible to find a pair of oppositely charged monomers $i$ and $j$, belonging, respectively, to $A$ and $B$, lying within a distance $r_{i j}<r_{\text {cont }}=1.2 \sigma$ from each other, where $r_{\text {cont }}$ has been chosen as a reasonable distance to yield a sufficient binding strength. This value is only slightly higher than both the distance at which our WCA potential goes to zero (i.e., $r_{\text {cut }}=2^{\frac{1}{6}} \sigma$, see Equation 1) and the distances at which we observe the main peaks in the pair distribution functions calculated between oppositely charged monomers at the free-swelling equilibrium (see Figure S9Pair distribution function calculated between positively and negatively charged monomers for polyelectrolytes with different $\Omega$ values. $r_{i j}$ is the distance between the pairs. The grey dotted vertical line indicates the value of the cutoff radius $r_{\text {cont }}$ used to define a "contact" (i.e., an ionic bond) between two oppositely charged stars (or arms).figure.caption.15). Despite the arbitrariness of the choice, we would like to stress that we explored alternative cutoff values, noticing that trends and behaviours which we report it this work stay preserved, and only small quantitative differences appeared.

Figure 5 shows the temporal evolution of $\left\langle\eta_{\mathrm{s}}\right\rangle$ for system with different $\Omega$ values. Due to our initial set-up, all curves for $t=0$ start at $\left\langle\eta_{\mathrm{s}}\right\rangle=1$ (see the inset in Figure 5), as one would expect in the presence of a solution of well-separated dimers in which each positively charged star is in contact with only one negative star, and vice versa. For $\Omega=2$, we notice 
that $\left\langle\eta_{\mathrm{s}}\right\rangle$ quickly drops to $\sim 0.4$, meaning that more than the $25 \%$ of the dimers dissociate, evidencing that the ionic bond are not strong enough to balance the entropic forces of the stars that want to achieve a homogenous distribution; hence, for $\Omega=2$ the loss of contacts is "overcompensated" by the increase in system entropy due to dimers dissociation. For $\Omega=3$, 4 , and 5 , instead, $\left\langle\eta_{\mathrm{s}}\right\rangle$ initially rapidly increases with $t$ and then it stabilizes around a value roughly equal to $1.4,2.3$, and 2.8 , respectively, which means that clusters containing more than 2 stars start to form due to higher order multipole attraction. Moreover, we observe that the time needed to reach equilibrium increases with $\Omega$, which is probably due to the progressively increase of the interaction strength between two stars bonded in a dimer, which in turn results in a higher potential barrier that must be overcome to break the ionic bonds apart.

\subsection{System structural properties at the free-swelling equilibrium}

In this section we discuss the structural properties of the supramolecular aggregates observed at the free-swelling equilibrium (see Figure 3), with a particular focus on investigating the presence of percolating networks as function of $\Omega$. Similarly to the number of contacts for a given star $A, \eta_{\mathrm{s}, A}$, one can also define the number of contacts formed by an individual $\operatorname{arm} \alpha, \eta_{\mathrm{a}, \alpha}$, and, consequently, the average value over all the chains in solution, $\left\langle\eta_{\mathrm{a}}\right\rangle$. To do this, we use the same definition of "contact" introduced previously, that is two oppositely charged arms results linked if exist at least one pair of oppositely charged monomers lying at a distance which is less than the cutoff radius $r_{\text {cont }}=1.2 \sigma$. From such a definition it immediately follows that if two arms are in contact, then so are the stars to which they belong. Figure 6 displays an example of the calculation of such properties for an aggregate composed of 3 stars. Especially in the case of lower $\Omega$ values, a non-negligible fraction of stars (and, consequently, arms) may posses, at least transiently, no contacts in solution. Thus, we define an "isolated" star as a polyelectrolyte star $A$ for which $\eta_{\mathrm{s}, A}=0$, and the average fraction of those as $\Delta_{\mathrm{s}}$. There will also be arms that do not have an ionic bond to 
any other arm, which we call in the following "dangling" arms, whose fraction is denoted by $\Delta_{\mathrm{a}}$. These are chains $\alpha$ for which $\eta_{\mathrm{a}, \alpha}=0$ (see Figure 6). Our results for the above discussed structural properties obtained for all systems are summarized in Table 1.

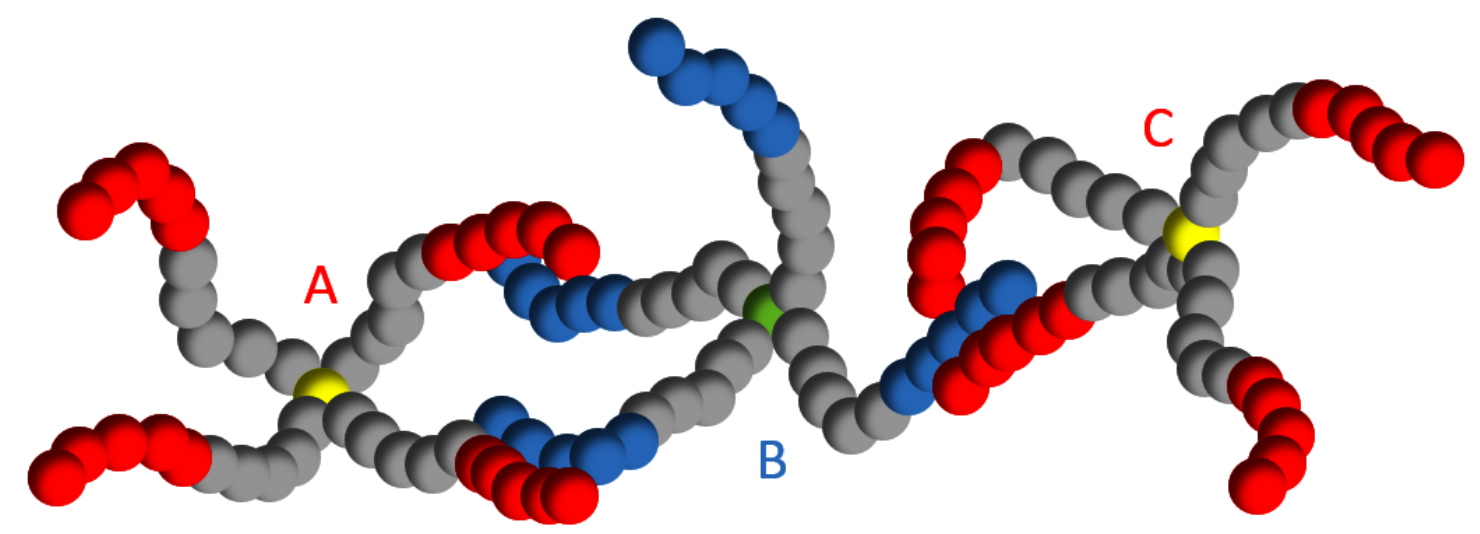

Figure 6: The picture represents a cluster of stars composed by 3 polyelectrolyte stars (i.e., $\mathscr{C}_{\mathrm{s}}=3$ ), $A, B$ and $C(\Omega=5)$ as an example. $A: \eta_{\mathrm{s}, A}=1, \sum_{\alpha=1}^{4} \eta_{\mathrm{a}, \alpha}=2,2$ dangling arms; $B: \eta_{\mathrm{s}, B}=2$, $\sum_{\beta=1}^{4} \eta_{\mathrm{a}, \beta}=4,1$ dangling arm; $C: \eta_{\mathrm{s}, C}=1, \sum_{\gamma=1}^{4} \eta_{\mathrm{a}, \gamma}=2,2$ dangling arms. Here, $\alpha, \beta$ and $\gamma$ are indexes that run over the four arms of $A, B$ and $C$, respectively. For this specific system, we find $\left\langle\eta_{\mathrm{s}}\right\rangle=\frac{4}{3} \simeq 1.33,\left\langle\eta_{\mathrm{a}}\right\rangle=\frac{4}{12} \simeq 0.33, \Delta_{\mathrm{s}}=0$ (no isolated stars), and $\Delta_{\mathrm{a}}=\frac{5}{2} \simeq 0.42$. The color scheme is the same as in Figure 3.

Table 1: Table summarizing results for the structural properties of the system at the free-swelling equilibrium. The numbers in brackets indicate the statistical error in the last significant digit.

\begin{tabular}{ccccc}
$\Omega$ & $\mathbf{2}$ & $\mathbf{3}$ & $\mathbf{4}$ & $\mathbf{5}$ \\
\hline \hline$L_{\text {eq }}(\sigma)$ & 36.07 & 28.27 & 25.65 & 23.84 \\
$C_{\mathrm{s}, \text { eq }}(\mathrm{mol} / \mathrm{l})$ & $2.55 \cdot 10^{-2}$ & $5.40 \cdot 10^{-2}$ & $7.05 \cdot 10^{-2}$ & $8.75 \cdot 10^{-2}$ \\
$\Delta_{\mathrm{a}}$ & $0.872(0)$ & $0.553(0)$ & $0.291(0)$ & $0.141(0)$ \\
$\Delta_{\mathrm{s}}$ & $0.575(1)$ & $0.091(0)$ & $0.006(0)$ & $0.0003(0)$ \\
$\left\langle\eta_{\mathrm{a}}\right\rangle$ & $0.130(0)$ & $0.481(0)$ & $0.857(1)$ & $1.21(0)$ \\
$\left\langle\eta_{\mathrm{a}}^{\prime}\right\rangle$ & $1.01(0)$ & $1.08(0)$ & $1.21(0)$ & $1.41(0)$ \\
$\left\langle\eta_{\mathrm{s}}\right\rangle$ & $0.500(1)$ & $1.74(0)$ & $2.89(0)$ & $3.82(1)$ \\
$\left\langle\eta_{\mathrm{s}}^{\prime}\right\rangle$ & $1.77(0)$ & $1.92(0)$ & $2.92(0)$ & $3.82(1)$ \\
\hline
\end{tabular}

Figure 7 (upper panel) shows the behaviour of $\left\langle\eta_{\mathrm{s}}\right\rangle$ and $\left\langle\eta_{\mathrm{a}}\right\rangle$ as a function of $\Omega$ at the free-swelling equilibrium. We notice that both observables scale linearly with the number of terminal charges. The increase in the number of contacts with $\Omega$ is due to the increased Coulomb attraction between the oppositely charged terminal end groups, which in turn 
results in a higher density (and, hence, in a lower mean star-star distance) at free-swelling equilibrium (see Table 1 and Figure S2Volume averaged virial pressure $P$ (bar) as a function of species concentration $C_{\mathrm{S}}(\mathrm{mol} / \mathrm{L})$ for the four $\Omega$ values. The dotted grey line is only a guide for the eye for discern positive and negative pressure values. Standard error bars are smaller than plot symbols where they're not visible.figure.caption.7).

Figure 7 (lower panel) also shows the fraction of isolated stars and dangling arms $\left(\Delta_{\mathrm{s}}\right.$ and $\Delta_{\mathrm{a}}$, respectively). As expected, both quantities decreases as $\Omega$ increases. For $\Omega=2$, more than 3 arms out of $4(\sim 87 \%)$ result in no electrostatic bonding to other chains, evidencing that we are not in presence of a network phase. We also observe a very high $\Delta_{\mathrm{a}}$ value for $\Omega=3(\sim 55 \%)$. This is in agreement with the observations that in this case each stars is on average connected with only 1.74 oppositely charged stars, and each arm possesses on average only $\sim 0.5$ contacts (see Table 1 ). Moving to the higher values of $\Omega=4$ and 5 , the fraction of dangling chains further decreases, but it remains, interestingly, higher than zero ( $\Delta_{\mathrm{a}} \simeq 0.29$ and 0.14 for $\Omega=4$ and 5 , respectively). However, for these large values of $\Omega$ almost all stars participate in forming ionic bonds, hence clusters, and $\Delta_{\mathrm{s}}$ is approximately 0 .

Nevertheless, we should be careful in interpreting the number of contacts provided by $\left\langle\eta_{\mathrm{a}}\right\rangle$ and $\left\langle\eta_{\mathrm{s}}\right\rangle$, since $\Delta_{\mathrm{a}}$ and $\Delta_{\mathrm{s}}$ show non-negligible values in the most of the analysed cases, hence the number of real contacts for non-isolated stars or and non-dangling arms is much larger. Therefore we recompute the averages of $\eta_{\mathrm{s}, A}$ and $\eta_{\mathrm{a}, \alpha}$ on the ensemble of stars $A$ and arms $\alpha$, respectively, that are involved in at least one contact (i.e., excluding from the averages all the isolated stars and all the dangling arms); we call these new observables $\left\langle\eta_{\mathrm{s}}^{\prime}\right\rangle$ and $\left\langle\eta_{\mathrm{a}}^{\prime}\right\rangle$, respectively. The renormalized contact data are contained in Table 1.

For $\left\langle\eta_{\mathrm{a}}^{\prime}\right\rangle$ we found that the probability for a chain to bind more than one arm increases with $\Omega$, varying from $1.01(\Omega=2)$ to $1.41(\Omega=5)$. Thus, when $\Omega$ is large, the possibility for an individual star's ionic block to get in contact with two (or even more) oppositely charged blocks is higher; the latter arms can either belong to the same star or to different 
ones. The fact that $\left\langle\eta_{\mathrm{a}}^{\prime}\right\rangle$ increases with $\Omega$ can be explained bearing in mind that increasing $\Omega$ results in: (i) an increased electrostatic attraction between the chains; (ii) a higher density of the system; and (iii) an increase in the size of the terminal charged blocks of an arm, and, hence, in an enhanced ability to accommodate two (or even more) oppositely charged chains, without the latter being in contact with each other. As for $\left\langle\eta_{\mathrm{s}}^{\prime}\right\rangle$, we notice that for the $\Omega=2$ and 3 cases, non-isolated polyelectrolytes tend to bind on average roughly 2 stars, whereas for $\Omega \geq 4$ our results do not considerably differ from those calculated including also the isolated stars in the averages, and this is obviously due to the negligible fraction of isolated stars found in solution.

To gain more insight into the architecture of supramolecular aggregates in solution, and in order to investigate if the systems percolate, we now look at the size distribution of aggregates composed by stars in solution. We define a "cluster of stars" (CoS) as the set of stars fulfilling the criterion that any of them is in contact with at least one other star that belong to such a CoS. Furthermore, we define the size $\mathscr{C}_{\mathrm{s}}$ of a CoS as the number of stars that belong to it (it follows that, e.g., dimers are $\operatorname{CoS}$ of size $\mathscr{C}_{\mathrm{s}}=2$ ).

Figure 8 shows the probability density for a given star to belong to a CoS of size $\mathscr{C}_{\text {s }}$ at the free-swelling equilibrium. This corresponds to the probability density to find a CoS with a certain size $\mathscr{C}_{\mathrm{s}}$ in solution weighted by the size itself and renormalized (the latter is provided in Figure S10Probability density to find a CoA with a certain size $\mathscr{C}_{\mathrm{s}}$ in solution for the four $\Omega$ values.figure.caption.16 in the ESI). For $\Omega=2$ we observe that most of the stars are bonded in dimers, the probability to find CoS composed by a higher number of polyelectrolyte stars decreasing with $\mathscr{C}_{\mathrm{s}}$, with only a few occurrences observed for $\mathscr{C}_{\mathrm{s}} \geq 6$. Let us also recall that for $\Omega=2$ roughly $58 \%$ of the stars are isolated in solution. Instead, for $\Omega=3$ the majority of the stars belong to the same large CoS, the size of the latter varying approximatively in the range $\mathscr{C}_{\mathrm{s}}=30-64$ (see the large peak which presents a maximum at $\mathscr{C}_{\mathrm{s}} \simeq 53$ ), with non-negligible number of isolated polyelectrolyte stars (see lower panel of Figure 7) or belonging to very small CoS. For the systems with $\Omega=4$ and 5 , the vast 

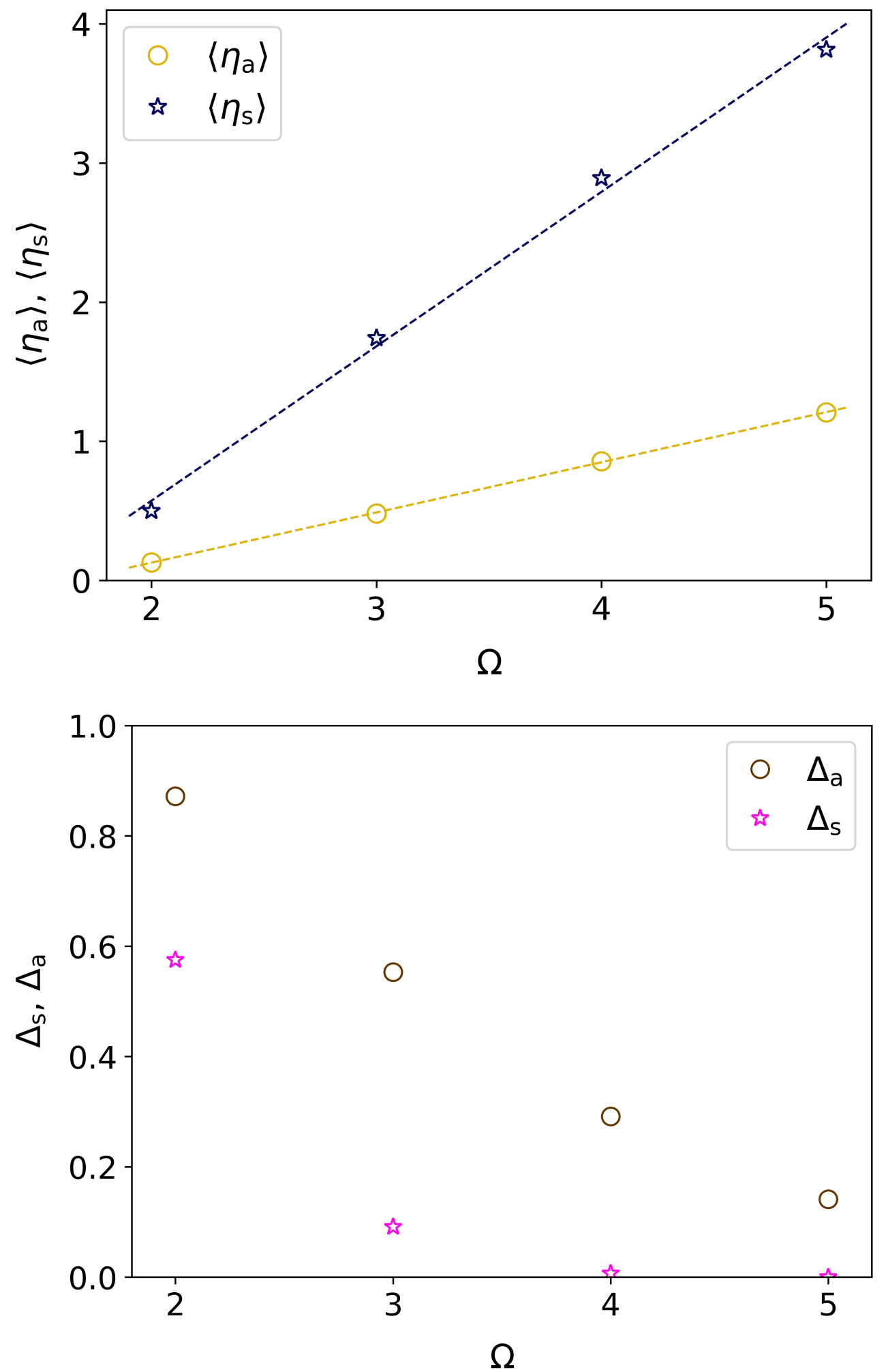

Figure 7: Upper panel: number of average contacts $\left\langle\eta_{\mathrm{a}}\right\rangle$ and $\left\langle\eta_{\mathrm{s}}\right\rangle$ as a function of $\Omega$; lower panel: fraction of dangling arms $\Delta_{\mathrm{a}}$ and isolated stars $\Delta_{\mathrm{S}}$ as a function of $\Omega$. Dashed lines in the upper panel represent linear fittings. Standard error bars are smaller than plot symbols. 


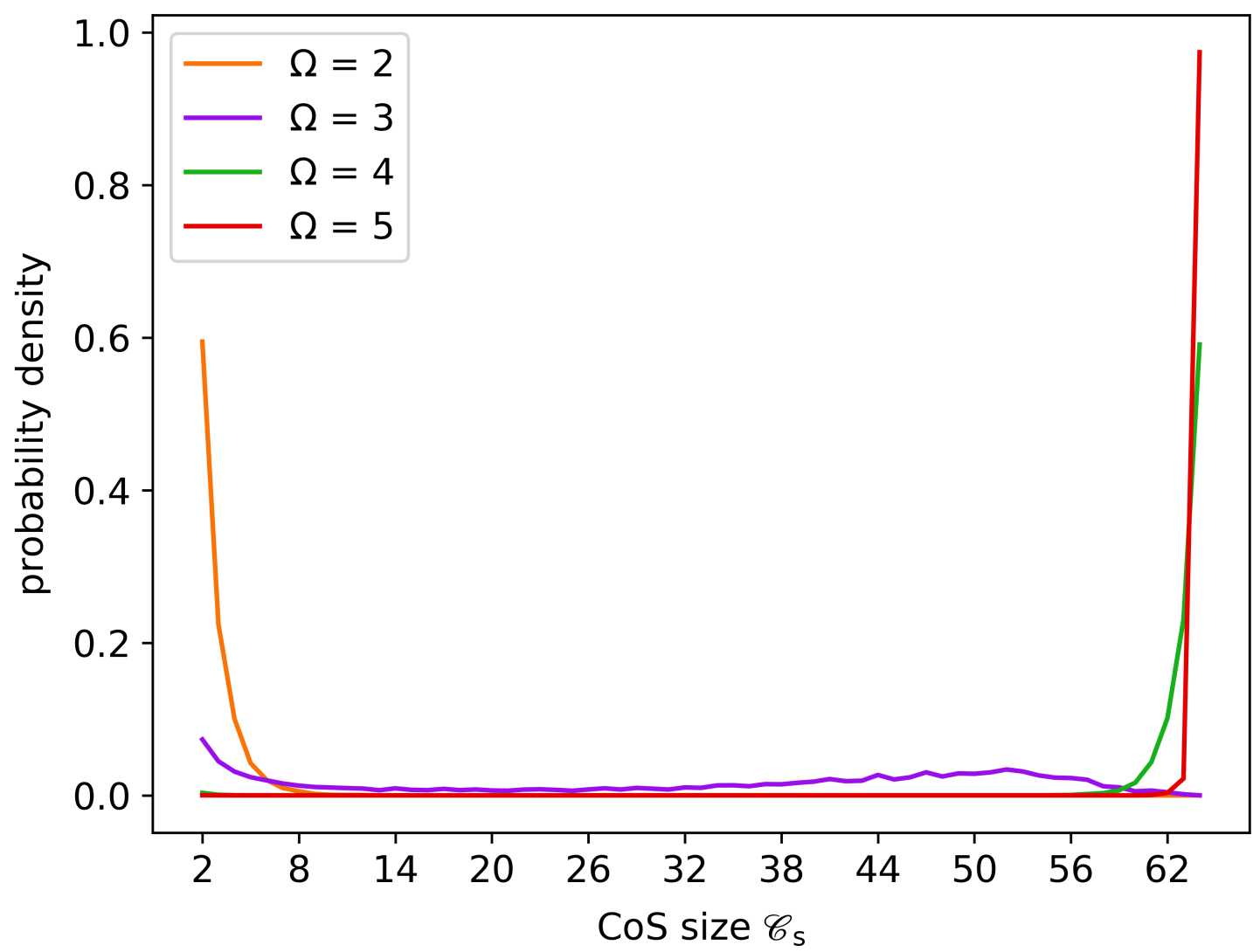

Figure 8: Probability density to find a given star in solution that belong to a CoS of size $\mathscr{C}_{\mathrm{s}}$. 
majority of stars belong to a single very large CoS, with almost no isolated polyelectrolyte stars $(\Omega=5)$ or only a few ones $(\Omega=4)$. Supported by a visual inspection of the snapshots for the $\Omega=4$ and 5 cases (see Figure 3, and see also the movies provided in the ESI), indicating that the box is completely filled by our polyelectrolyte stars, and also recalling the high number of contacts observed (see the upper panel of Figure 7), we are confident that we are observing a percolating gel.

In analogy with what was done for CoS, one can identify clusters formed by interacting individual arms. We define a "cluster of arms" (CoA) of size $\mathscr{C}_{\mathrm{a}}$ as the set of $\mathscr{C}_{\mathrm{a}}$ chains that fulfils the criterion that any of them is in contact with at least one other chain that belongs to the same CoA (definition very similar to the one used by Gârlea et al. in their work about the self-organization of soft patchy colloids ${ }^{34}$ ). Before discussing the results, let us stress here that the size of a CoA is not equal to the number of arms involved in a bond, and this is due to the fact that two chains belonging to the same CoA may not be in contact, but rather be far from each other; this is true especially for the systems with $\Omega=4$ and 5 , for which the charged block of each arm is "long" enough to accommodate more than one oppositely charged chain (we provide a pictorial illustration in the right panel of Figure 9).

The plot in Figure 9 shows CoA size distributions for all our simulated $\Omega$ values. When $\Omega$ is low, the vast majority of arms is involved in the formation of simple positive-negative contacts, or are dangling, whereas for higher values $\Omega$ values the CoA mean size increases, and we observe for $\Omega=4$ and 5 that there is a non-zero probability to find CoA formed by 6 or more chains.

Since a CoA may not be charge-neutral we provide in Figure 10 heatmaps that show the probability density to find a CoA with a certain size $\mathscr{C}_{\mathrm{a}}$ and an "excess of arms" $\mathscr{Q}_{\text {a }}$ in solution. We define $\mathscr{Q}_{\mathrm{a}}$ as the excess charge carried by a CoA divided by $\Omega$. At first glance, in all the panels we notice a very marked checkboard pattern; the latter is due to the fact that a CoA composed by an even (odd) number of arms must necessarily show an even (odd) $\mathscr{Q}_{\text {a }}$. A symmetry shown in all the panels is that for a certain value of $\mathscr{Q}_{\text {a }}$ the probability 

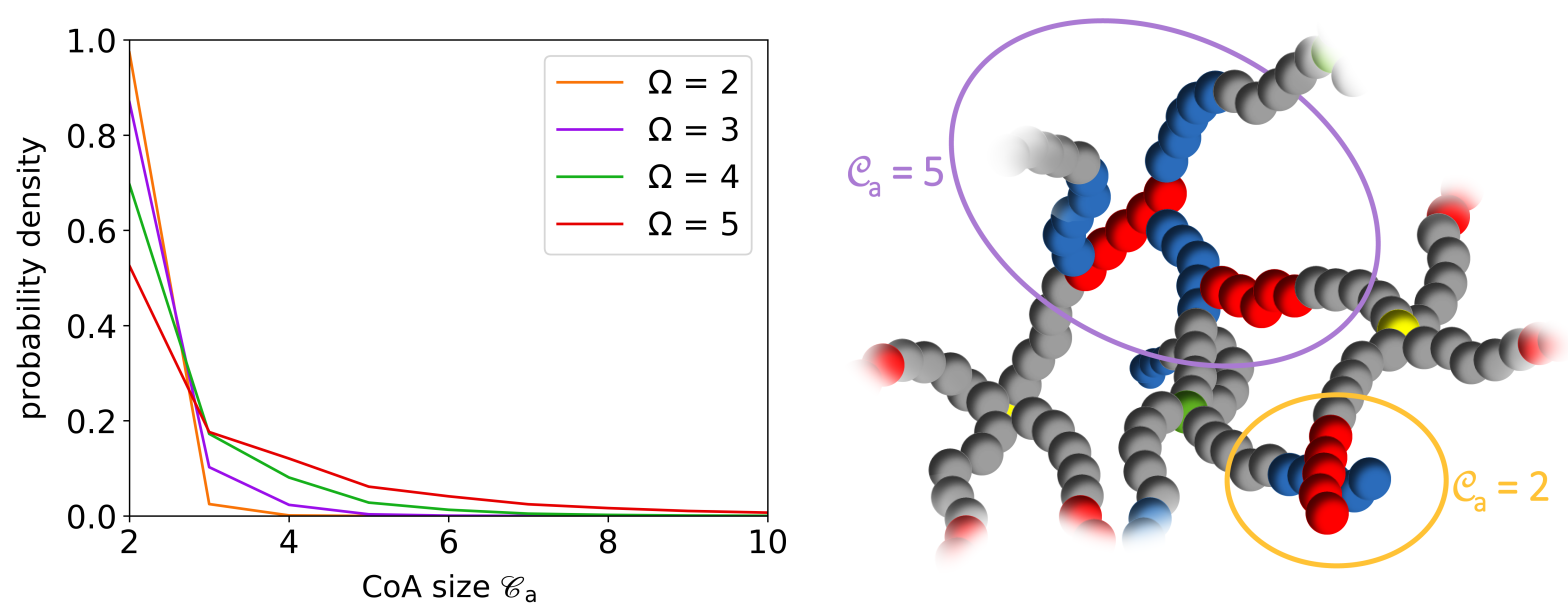

Figure 9: Left panel: CoA size distribution for the four $\Omega$ values. Right panel: examples of clusters formed by 5 (i.e., $\mathscr{C}_{\mathrm{a}}=5$, circled in violet) and 2 (i.e., $\mathscr{C}_{\mathrm{a}}=2$, circled in orange) arms. For these CoA, the "excess of arms" are $\mathscr{Q}_{\mathrm{a}}=-1$ and 0 , respectively. Notice that (i) arms that belong to the same CoA may not be directly in contact with each other, and (ii) arms that are tethered to the same central bead may belong to different CoA. The colour scheme is the same as in Figure 3.

density to observe $-\mathscr{Q}_{\mathrm{a}}$ is almost identical (as it should be by the global symmetry of our monodispersed systems); we take this as another indication that our simulations sampled the phase space properly.

From the heatmaps we observe that, for a given size $\mathscr{C}_{a}$, the probability density decreases as $\left|\mathscr{Q}_{\mathrm{a}}\right|$ increases. For $\Omega=2$ (top left panel of Figure 10) we observe that most of the arms are involved in the simplest type of bond, that is, a positive-negative contact (hence, $\mathscr{Q}_{\mathrm{a}}=0$ ), with the probability to find contacts involving 3 chains being roughly two order of magnitude smaller. Nevertheless, it seems more probable to find a CoA with $\mathscr{C}_{\mathrm{a}}=3$ and $\left|\mathscr{Q}_{\mathrm{a}}\right|=1$, with respect to a neutral CoA in which 4 chains are involved. As $\Omega$ increases, we can also find larger sizes of CoA probably due to the ability of the large charge patches arms to bind to more than one oppositely charged chain. Furthermore, we observe several occurrences of CoA composed by a very large number of chains especially for $\Omega=5$ (up to 44, see Figure $\mathrm{S} 11-\log _{10}$ of the probability density to observe a chain belonging to a CoA of size $\mathscr{C}_{\mathrm{a}}$ for $\Omega=4$ and 5 . In case of 0 occurrences of clusters with a certain size $\mathscr{C}_{\text {a }}$, we arbitrary set the $-\log _{10}$ of the probability density to 5.6.figure.caption.17). 
As a final comment, let us point out that the calculated sizes of both CoS and CoA could be slightly underestimated due to the fact that we never counted (i) two positive (or two negative) polyelectrolytes, or (ii) two stars that are in touch only via neutral monomers, as being in contact; although those situations seem to be very unlikely, they may occur due to thermal fluctuations especially in the case of low $\Omega$ values, or when the system density is very high.
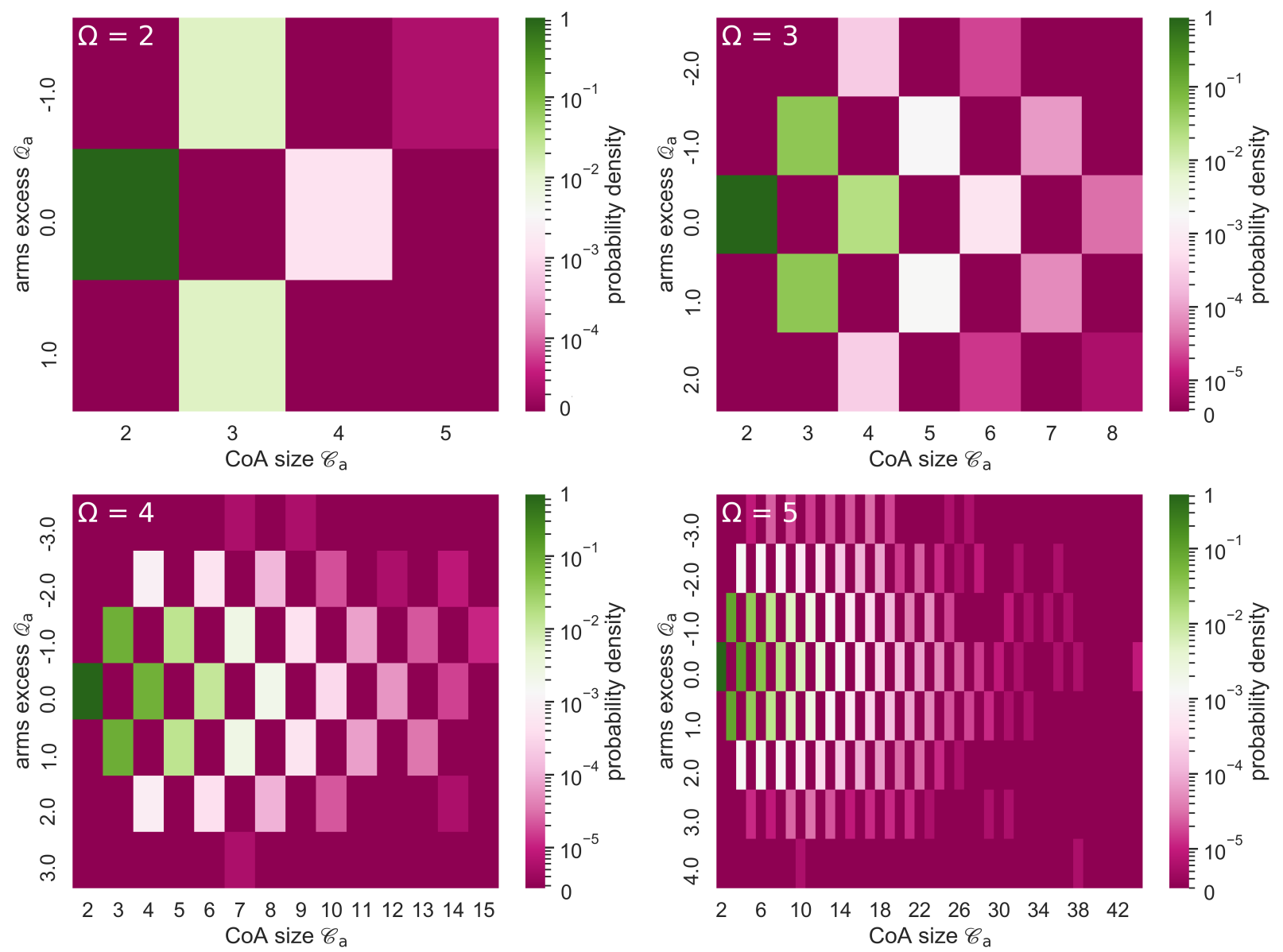

Figure 10: Heatmaps showing the probability density to find a CoA with a given size $\mathscr{C}_{\text {a }}$ and an excess of charges $\mathscr{Q}_{\mathrm{a}}$ for $\Omega=2,3,4$, and 5 .

\subsection{Ionic Bond Lifetimes}

A visual inspection of the movies provided in the ESI suggests that even the ionic bonds formed with $\Omega=5$ are quite soft and allow for a continuous restructuring of the network. 
In order to gain a more quantitative understanding of the bond strengths and lifetimes we preformed an analysis of the bond dynamics in time and investigated which mechanisms can lead to the breaking and subsequent reformation of such contacts.

We begin by defining a "contact time" $\tau$ as the time a contact between two arms $\alpha$ and $\beta$ persists in solution. The upper panel of Figure 11 shows the probability density $p(\tau)$ to observe a contact that breaks apart in the interval $[\tau ; \tau+\Delta \tau]$, with $\Delta \tau=0.05$. Contrary to the exponential decay expected, we notice that $p(\tau)$ displays a maximum around $\tau \simeq 0.3$ for any $\Omega$. As previously discussed by Bunker and Hase ${ }^{58}$, this is a consequence of the "initial state selection" that derives from the orientation of the relative linear momentum for the two arms that have just come closer to be identified as a contact. Dissociation of the newly formed bond may, in fact, require some time to partially invert their relative velocity so that they can "wander back" to a distance at which the bond can be considered broken. Most likely, a properly oriented collision between the two approaching arms is needed to trigger such partial inversion, so that some degree of ballistic (hence, non-statistical) behaviour may be present during the initial stages of the process. This notwithstanding, mean lifetimes $\left(\bar{\tau}_{\text {calc }}=\int_{0}^{\infty} \tau p(\tau) d \tau\right)$ have been computed with the shown $p(\tau)$, and these are $0.40,0.50$, 0.60 , and 0.68 for $\Omega=2,3,4$, and 5 , respectively (see Table 2 for a summary). As one could have expected, the mean lifetime increase with $\Omega$.

A mono-molecular event is formally described by the equation $\alpha \cdots \beta \longrightarrow \alpha+\beta$, where "..." denotes a contact between two arms. As $p(\tau)$ deviates from the statistical behavior expected from such an event, also the fraction of surviving contacts $\left(N(t) / N_{0}\right)$ versus time should deviate from the exponential decay law $e^{-t k_{1}}=e^{-t / \bar{\tau}_{1}}$ that are typical for such processes, where $k_{1}$ and $\tau_{1}$ are the first-order rate constant and the corresponding mean lifetime. To prove this point we investigated the short time behavior of $N(t) / N_{0}$ (see the inset in the lower panel of Figure 11). At short times the lack of linearity displayed by $\ln \left[N(t) / N_{0}\right]$ shows a faster decay, indicating the presence of an intrinsically non-statistical (as in Rice-RamspergerKassel-Marcus theory ${ }^{59,60}$ ) behavior for the dissociation process. This is characterized by 

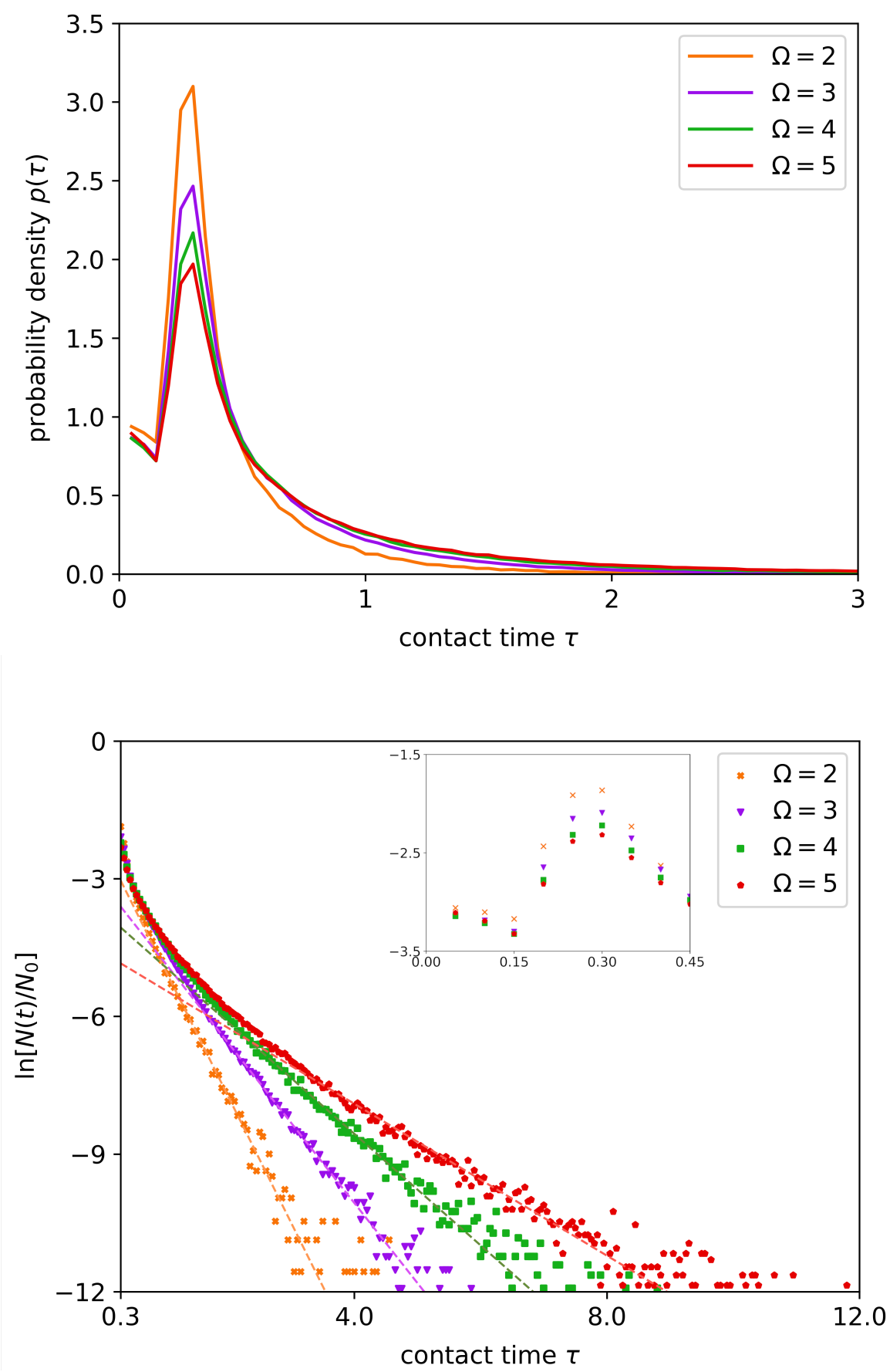

Figure 11: Upper panel: probability density $p(\tau)$ to observe a contact persisting in solution for a time $\tau$. Natural logarithm of $N(t) / N_{0}$ versus the contact time $\tau$; here, dashed lines represent the linear fittings of the statistical part. The inset shows the short time behaviour of $\ln \left[N(t) / N_{0}\right]$ versus $\tau$. $\tau$ is in system time units. 
an elevate population of fast dissociating states that are generated right after contact formation, leading to a high number of short time dissociation events. At longer times, $N(t) / N_{0}$ instead appears to decay exponentially, so that fitting the long time part of the scatter plot allowed us to compute the statistically derived mean lifetimes $\tau_{1}$, which are $0.36,0.57,0.82$, and 1.20 for $\Omega=2,3,4$, and 5 , respectively. As one would have expected, $\tau_{1}>\bar{\tau}_{\text {calc }}$ for $\Omega \geq 3$, as the fitting process eliminates the majority of fast dissociation events. The fact that such inequality is not satisfied when $\Omega=2$ is simply due to the limited sample of events collected and the related inaccuracy of the long time distribution. Also, the increase of the ratio $\tau_{1} / \bar{\tau}_{\text {calc }}$ upon increasing $\Omega$ seems to support the idea that the collision between two arms forming a contact are the cause of the high population of fast dissociating dimers. In fact, it is well known that it becomes increasingly less likely for a colliding pair to redistribute into internal modes a sufficiently large fraction of their relative kinetic energy so to allow the formation of a meta-stable dimer the higher the kinetic energy is ${ }^{61}$. Obviously, the latter increases upon increasing $\Omega$ due to stronger Coulomb interactions.

Table 2: Contact times (in system time units) and relative frequencies for the three mechanisms $(\mathcal{I}$, $\left.\mathcal{S}_{\text {ant }}, \mathcal{S}_{\text {pos }}\right)$ and the non-classifiable ones $(\mathcal{O}) . E_{\text {tot }}$ is the relative total number of events observed calculated with respect to the $\Omega=2$ case. The numbers in brackets indicate the statistical error on the last significant digit.

\begin{tabular}{ccccc}
$\Omega$ & $\mathbf{2}$ & $\mathbf{3}$ & $\mathbf{4}$ & $\mathbf{5}$ \\
\hline \hline $\bar{\tau}_{\text {calc }}$ & $0.401(0)$ & $0.504(1)$ & $0.601(1)$ & $0.676(0)$ \\
$\tau_{1}$ & $0.362(1)$ & $0.573(9)$ & $0.823(9)$ & $1.20(1)$ \\
$\mathcal{I}$ & $79.7(1) \%$ & $68.3(1) \%$ & $52.3(1) \%$ & $41.1(1) \%$ \\
$\mathcal{S}_{\text {ant }}$ & $1.3(0) \%$ & $5.5(0) \%$ & $10.2(1) \%$ & $12.1(0) \%$ \\
$\mathcal{S}_{\text {pos }}$ & $17.1(1) \%$ & $17.9(1) \%$ & $20.6(0) \%$ & $21.2(0) \%$ \\
$\mathcal{O}$ & $1.9(0) \%$ & $8.2(1) \%$ & $16.9(0) \%$ & $24.8(1) \%$ \\
$E_{\text {tot }}$ & 1.00 & 2.91 & 4.34 & 5.44 \\
\hline
\end{tabular}

In order to better analyse the details of the discussed non-statistical effects and to investigate which other mechanism may be involved in restructuring the network of electrostatic bonds, we computed the relative frequency of three different mechanisms by means of which an arm $\alpha$ can loose a contact (that lasted for a time $t=\tau_{\text {bond }}$ ) with an oppositely charged 
$\operatorname{arm} \beta$, and switch the latter with a new arm $\gamma$ after some time $\tau_{\text {lag. }}$. These three mechanisms are illustrated in Figure 12, and they are:

"intermittent bond" $(\mathcal{I})$ : a contact temporarily breaks reforming after a time $\tau_{\text {lag }}$ has elapsed (see Figure 12 (a))

e.g., $\alpha \cdots \beta \underset{\tau_{\text {lag }}}{\stackrel{\tau_{\text {bond }}}{\rightleftarrows}} \alpha+\beta$.

"anticipated partner switch" ( $\left.\mathcal{S}_{\text {ant }}\right)$ : a negative (positive) arm $\gamma$ binds to an existing contact causing the detachment of another negative (positive) chain (see Figure 12 (b))

e.g., $\gamma+\alpha \cdots \beta \stackrel{\tau_{\text {lag }}}{\longrightarrow} \gamma \cdots \alpha \cdots \beta \stackrel{\tau_{\text {bond }}}{\longrightarrow} \gamma \cdots \alpha+\beta$.

"postponed partner switch" $\left(\mathcal{S}_{\mathrm{pos}}\right)$ : an arm $\alpha$ loses a contact and then form a new bond with a different chain after a time $\tau_{\text {lag }}$ (see Figure $12(\mathrm{c})$ ) e.g., $\gamma+\alpha \cdots \beta \stackrel{\tau_{\text {bond }}}{\longrightarrow} \gamma+\alpha+\beta \stackrel{\tau_{\text {lag }}}{\longrightarrow} \gamma \cdots \alpha+\beta$.

Additionally, we classify all those mechanisms that cannot be included in the mentioned categories as "non-classifiable mechanisms" $(\mathcal{O})$. A more detailed discussion on the three mechanisms and the protocols implemented to categorize dissociation events is provided in the ESI.

Figure 13 and Table 2 show the results of our analysis. In the latter, we also report the relative frequency of events observed, $E_{\text {tot }}$, with respect to the $\Omega=2$ case. We notice that $E_{\text {tot }}$ increases with $\Omega$, which is probably related to the increase of the equilibrium density with $\Omega$. For $\Omega=2$, the vast majority of events $(\sim 80 \%$, see Table 2$)$ are classified as "intermittent contacts", which is probably due to the fact that stars are assembled in dimers or small CoS, so that mechanisms involving the exchange of arms are relatively rare, whereas temporary detachment due to thermal fluctuations are favoured by the relatively low Coulomb attraction between oppositely charged chains. Roughly $\sim 17 \%$ of the events result are "postponed partner switches", whereas the fraction of $\mathcal{S}_{\text {ant }}$ and $\mathcal{O}$ mechanisms results negligible. 

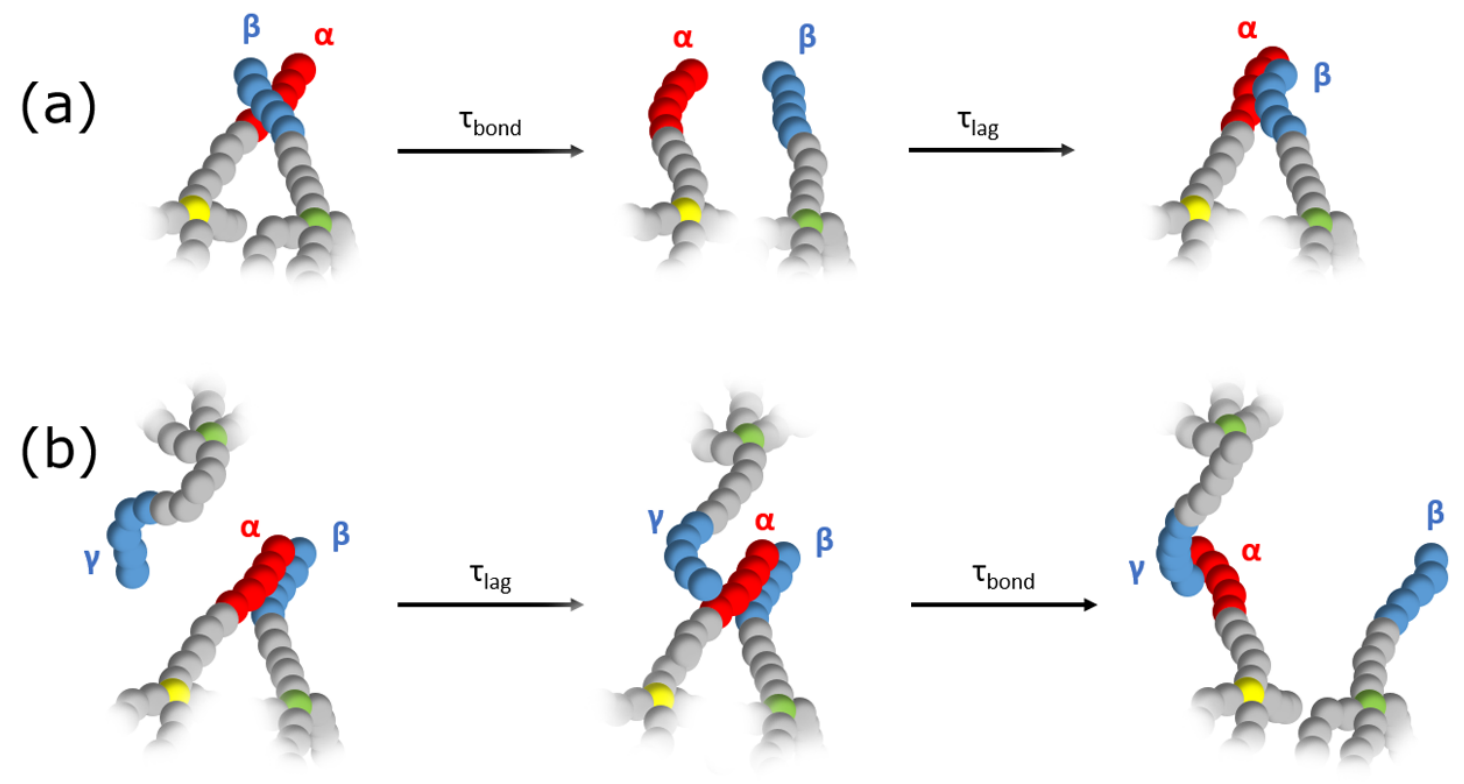

(c)

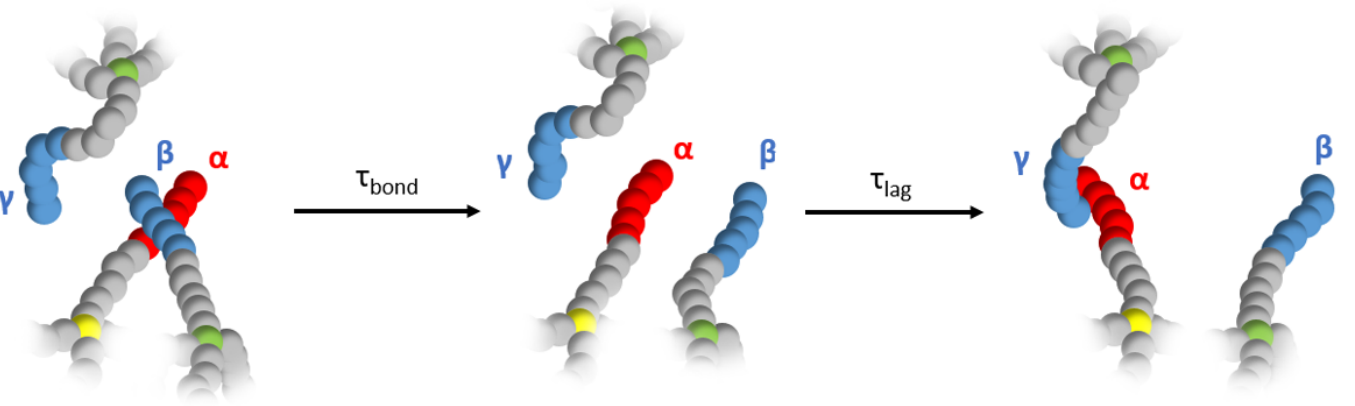

Figure 12: Pictorial description of the three mechanisms investigated: (a) $\mathcal{I}$; (b) $\mathcal{S}_{\text {ant }}$; (c) $\mathcal{S}_{\text {pos }}$. 
As $\Omega$ increases, we observe an enhancement in the fraction of $\mathcal{S}_{\text {pos }}, \mathcal{S}_{\text {ant }}$, and $\mathcal{O}$ mechanisms, a clear evidence of the possibility for the network bonds to restructure themselves. Again, the increase in the number of $\mathcal{S}_{\text {pos }}$ may be rationalized by recalling that the equilibrium density increases with $\Omega$; in fact, the denser the solution gets, the more likely it becomes for a positively (negatively) charged arm to replace a dissociating positive (negative) arm of a vicinal contact. As for the $\mathcal{S}_{\text {ant }}$ mechanism, also the increasing length of charged segments may play a role in increasing its frequency. The longer the charged block is, the higher is the probability for an arm $\alpha$ to "accommodate" more than one contact with oppositely charged segments $(\beta, \gamma$, etc.). This we attribute to the possibility for the charged portion of $\beta$ and $\gamma$ arms to stay further away from each other while being coordinated to the same $\alpha$ arm. Importantly, the $1: 2$ positive-negative (or vice versa) coordination mode is also expected to facilitate the detachment of, e.g., the $\beta$ arm, as its binding energy with $\alpha$ ought to be lower due to the repulsive Coulomb interaction with $\gamma$.

As for the non-statistical behavior previously discussed, Figure 13 presents the plots of $\ln \left[N(t) / N_{0}\right]$ versus $\tau_{\text {bond }}$ for all $\Omega$ values and the three discussed mechanism. From these, one notices that fast dissociation events arise mainly as a consequence of the $\mathcal{S}_{\text {pos }}$ and $\mathcal{I}$ mechanisms, the latter invariably being the most likely whereas the former presenting a more marked fractional deviation from the statistical behavior. Juxtaposing these results with similar ones concerning $\tau_{\text {lag }}$ (see Figure S13Natural logarithm of $N(t) / N_{0}$ versus $\tau_{\text {lag }}$ (system time units) for the three mechanisms and the four $\Omega$ values.figure.caption.20), it emerges that it takes more time for two free (e.g., $\alpha$ and $\beta$ ) arms to form a contact from a dissociated state than breaking an electrostatic bond already formed. Given the unhindered nature of the process forming a contact from dangling arms, we believe the previously discussed ballistic dynamics to be a robust justification for the non-statistical behavior evidenced by our data.

To conclude this section, we mention that the increase in the fraction of $\mathcal{O}$-events upon increasing $\Omega$ is mainly due to an increase in the number of events that present multiple ap- 
proaches or detachments taking place contemporary within the time window represented by our time resolution $(\Delta \tau=0.05)$. This is well supported by Figure S12Fraction of non-classifiable mechanisms $(\mathcal{O})$ as a function of the time resolution $\Delta \tau$ for the $\Omega=5$ case.figure.caption.19, which shows the dependency of this fraction on $\Delta \tau$ itself for the case $\Omega=5$. Obviously, if $\Delta \tau$ was infinitely small, only single dissociation or association events would be recorded.
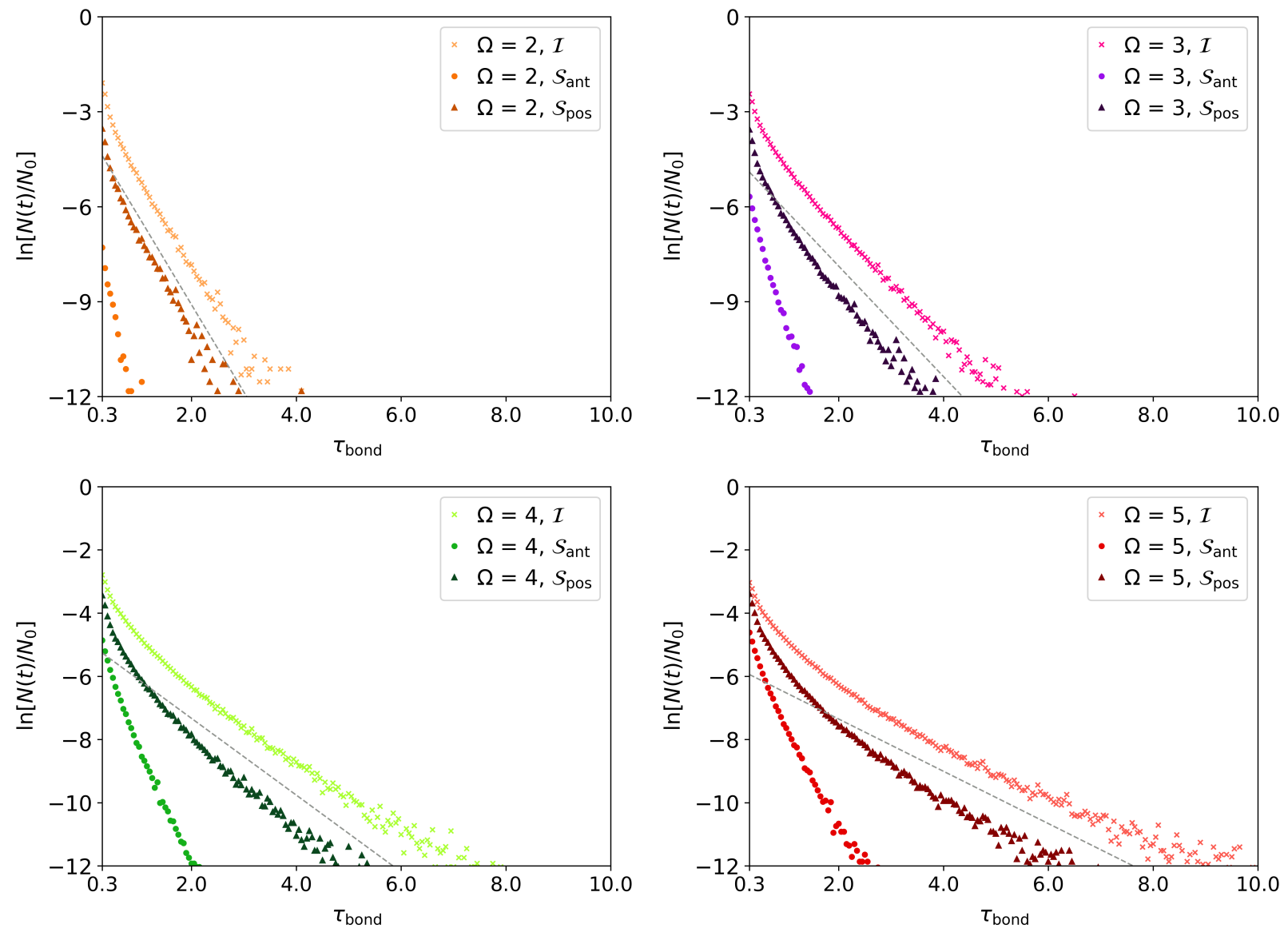

Figure 13: Natural logarithm of $N(t) / N_{0}$ versus $\tau_{\text {bond }}$ (system time units) for the three mechanisms and the four $\Omega$ values. We also report the linear fits (dashed grey lines) calculated in Figure 11 for a direct comparison. 


\section{Conclusions}

We performed a Langevin molecular dynamics computational study in order to investigate the possibility to create a supramolecular network by mixing equal amounts of oppositely charged di-block star-shaped polyelectrolytes under salt free conditions. Our polyelectrolyte stars consisted of four polymeric bead-spring chains tethered to a central common bead and carrying a tunable number $\Omega$ of (positively or negatively) charged monomers at their ends, using a polyelectrolyte primitive model in an implicit solvent ${ }^{62}$.

We investigated systems with $\Omega$ values from 1 to 5 at different concentrations and determined from the P-V curves the equilibrium concentration with respect to a pure water phase. Our results yielded equilibrium concentrations for $\Omega \geq 2$ (see Figure 2 and 3 ). The reproducibility of our results for three different simulations protocols (see Section 2.2 and Figure 4), and the fact that also a solution of pre-thermalized dimers has relaxed (Figure 5) demonstrate that our simulations yield true equilibrium structures.

The observed phases at free-swelling equilibrium have been characterized via many struc-

tural parameters (see Table 1 ). We found that the number of contacts increases with $\Omega$, with a non-zero probability to observe contacts involving more than two oppositely charged arms for $\Omega \geq 4$. Conversely, the fraction of dangling arms decreases with the number of terminal charges, but it remains interestingly non-zero even when $\Omega=5$, for which we observe that $\sim 14 \%$ of the arms are not involved in any ionic bond.

From an analysis of the cluster of stars $(\mathrm{CoS})$ in solution we found that for the $\Omega=2$ case only dimers and small oligomers can form, whereas for $\Omega=4$ and 5 all stars are part of a single macro-aggregate (see Figure 8), the latter being identified as a percolating network spanning the whole box, i.e. a gel-like phase, after a visual inspection of snapshots and trajectory movies provided in the ESI. The $\Omega=3$ case turned out to be an intermediate one, in which most of the stars belong to a main CoS (the latter fluctuating in size), with a non-negligible number of stars being isolated in solution or belonging to small oligomers or secondary CoS. An analogous analysis has been performed for clusters composed by 
individual arms (CoA), revealing that also in this case their size increases with $\Omega$ (see Figure 9). Furthermore, we found some CoA with an excess charge, whose probability of occurance increases with $\Omega$ as well. Overall, the ionic cross-links structure is far from being trivial even for the case of equal charged terminal bonds. The simple picture of having mostly saturated ionic bonds is definitely not applicable.

In order to gain more insight in the dynamical processes of forming and dissolving ionic bonds, we analysed the contact time of such bonds as function of $\Omega$. As expected, the mean lifetime increases with the latter. Nevertheless, we found strong deviations from the expected first-order dissociation kinetic, the latter underestimating the frequency of persistent contacts and fast dissociation events for almost all cases (see Table 2). We described various mechanism leading to contact formations and ruptures, observing a non-zero probability (even for low $\Omega$ values) for an arm involved in a bond to be replaced by another chain with the same charge, a finding that clearly opens up the possibility for the network to restructure itself in time.

This study has been so far restricted to a small parameter regime, most notably is the absence of counterions or background salt ions. As such it should serve as a proof-ofprinciple that gel formation in mixtures of oppositely charged polyelectrolyte stars is possible. However, more complicated situations like adding different ion types as mono- or even multivalent ions (e.g., in order to increase bond strengths by divalent ions bridging), changing the ionic block from being a strong polyelectrolyte to a weak polyelectrolyte and adding thus the possibility of the charged groups to respond to $\mathrm{pH}$ or to form inter-molecular charged hydrogen bonds ${ }^{63-65}$, or introducing polydispersity in arm length or in charged block length can easily be simulated with more refined models, and these systems are currently under investigation. The experimental realization of some of such systems is currently pursued in the group of F. H. Schacher 66 . 


\section{Acknowledgement}

MM thanks the Università degli Studi dell'Insubria for funding under the scheme "Fondo d'Ateneo per la Ricerca (FAR 2018). AT thanks Università degli Studi dell'Insubria for his Ph.D. studentship. The authors acknowledge inspiring discussions with P. Košovan, F. H. Schacher, D. Sean, and J. Zeman. This work was performed within the collaborative framework of the research unit Adaptive Polymer Gels with Controlled Network Structure (FOR2811), funded by the German Research Foundation under No. 423435431.

\section{Supporting Information Available}

In the Electronic Supporting Information we provide:

- additional graphs and trajectory snapshots supporting our results;

- a detailed description of the algorithm used to sample structural and dynamical system properties and to categorize ionic bonds restructuring mechanisms;

- trajectory movies showing both the entire simulation box and selected system details for various $\Omega$ values.

\section{References}

(1) Zohuriaan-Mehr, M. J.; Kabiri, K. Superabsorbent polymer materials: A review. Iranian Polymer Journal 2008, 17, 451-477.

(2) Peppas, N. A.; Bures, P.; Leobandung, W.; Ichikawa, H. Hydrogels in pharmaceutical formulations. European journal of pharmaceutics and biopharmaceutics 2000, 50, 2746.

(3) Samsonov, G. V.; Kuznetsova, N. P. Crosslinked Polyelectolytes in Biology. Advances in Polymer Science 1992, 104, 1-50. 
(4) Jia, X.; Kiick, K. L. Hybrid Multicomponent Hydrogels for Tissue Engineering. Macromolecular Bioscience 2009, 9, 140-156.

(5) Jagur-Grodzinski, J. Polymeric gels and hydrogels for biomedical and pharmaceutical applications. Polymers for Advanced Technologies 2009, 21, 27-47.

(6) Meenach, S.; Anderson, K.; Hilt, J. In Safety of Nanoparticles; Webster, T. J., Ed.; Nanostructure Science and Technology; Springer New York, 2009; pp 131-157.

(7) Tamura, H.; Furuike, T.; Nair, S.; Jayakumar, R. Biomedical applications of chitin hydrogel membranes and scaffolds. Carbohydrate Polymers 2011, 84, 820 - 824, Advances in chitin/chitosan science and their applications.

(8) Sannino, A.; Esposito, A.; Rosa, A. D.; Cozzolino, A.; Ambrosio, L.; Nicolais, L. Biomedical application of a superabsorbent hydrogel for body water elimination in the treatment of edemas. Journal of Biomedical Materials Research Part A 2003, 67A, 1016-1024.

(9) Zavan, B.; Cortivo, R.; Abatangelo, G. Hydrogels; Springer Milan, 2009; pp 1-8.

(10) Zohuriaan-Mehr, M. J.; Omidian, H.; Doroudiani, S.; Kabiri, K. Advances in nonhygienic applications of superabsorbent hydrogel materials. Journal of Materials Science 2010, 45, 5711-5735.

(11) Kazanskii, K.; Dubrovskii, S. Polyelectrolytes Hydrogels Chromatographic Materials. Advances in Polymer Science; Springer Verlag, 1992; Vol. 104; pp 97-133.

(12) Polyelectrolyte gels: Properties, Preparation, and Applications; ACS Symposium Series No. 480; American Chemical Society, Washington D.C., 1992.

(13) Höpfner, J.; Klein, C.; Wilhelm, M. A Novel Approach for the Desalination of Seawater by Means of Reusable Poly(acrylic acid) Hydrogels and Mechanical Force. Macromolecular Rapid Communications 2010, 31, 1337. 
(14) Höpfner, J.; Richter, T.; Košovan, P.; Holm, C.; Wilhelm, M. In Intelligent Hydrogels; Sadowski, G., Richtering, W., Eds.; Progress in Colloid and Polymer Science; Springer International Publishing, 2013; Vol. 140; pp 247-263.

(15) Richter, T.; Landsgesell, J.; Košovan, P.; Holm, C. On the efficiency of a hydrogel-based desalination cycle. Desalination 2017, 414, 28-34.

(16) Arens, L.; Barther, D.; Landsgesell, J.; Holm, C.; Wilhelm, M. Poly(sodium acrylate) hydrogels: synthesis of various network architectures, local molecular dynamics, salt partitioning, desalination and simulation. Soft Matter 2019, 15, 9949-9964.

(17) Kozlovskaya, V.; Kharlampieva, E.; Chang, S.; Muhlbauer, R.; Tsukruk, V. V. pHResponsive Layered Hydrogel Microcapsules as Gold Nanoreactors. Chemistry of Materials 2009, 21, 2158-2167.

(18) Mohan, Y. M.; Lee, K.; Premkumar, T.; Geckeler, K. E. Hydrogel networks as nanoreactors: A novel approach to silver nanoparticles for antibacterial applications. POLYMER 2007, 48, 158-164.

(19) Diaz, D. D.; Kuehbeck, D.; Koopmans, R. J. Stimuli-responsive gels as reaction vessels and reusable catalysts. Chemical Society Reviews 2011, 40, 427-448.

(20) Oh, J. K.; Drumright, R.; Siegwart, D. J.; Matyjaszewski, K. The development of microgels/nanogels for drug delivery applications. Progress in Polymer Science 2008, 33, 448-477.

(21) Hamidi, M.; Azadi, A.; Rafiei, P. Hydrogel nanoparticles in drug delivery. Advanced Drug Delivery Reviews 2008, 60, 1638-1649.

(22) Makino, K.; Idenuma, R.; Murakami, T.; Ohshima, H. Design of a rate- and timeprogramming drug release device using a hydrogel: pulsatile drug release from kappa- 
carrageenan hydrogel device by surface erosion of the hydrogel. Colloids and Surfaces B-Biointerfaces 2001, 20, 355-359.

(23) Tada, D.; Tanabe, T.; Tachibana, A.; Yamauchi, K. Drug release from hydrogel containing albumin as crosslinker. Journal of Bioscience and Bioengineering 2005, 100, $551-555$.

(24) Wang, Q.; Li, S.; Wang, Z.; Liu, H.; Li, C. Preparation and Characterization of a Positive Thermoresponsive Hydrogel for Drug Loading and Release. Journal of Applied Polymer Science 2009, 111, 1417-1425.

(25) Seiffert, S.; Sprakel, J. Physical chemistry of supramolecular polymer networks. Chemical Society Reviews 2012, 41, 909-930.

(26) Hoffman, A. S. Hydrogels for biomedical applications. Advanced Drug Delivery Reviews 2012, 64, 18-23.

(27) Rossow, T.; Habicht, A.; Seiffert, S. Relaxation and dynamics in transient polymer model networks. Macromolecules 2014, 47, 6473-6482.

(28) Seiffert, S. Origin of nanostructural inhomogeneity in polymer-network gels. Polymer Chemistry 2017, 8, 4472-4487.

(29) Borisova, O.; Billon, L.; Zaremski, M.; Grassl, B.; Bakaeva, Z.; Lapp, A.; Stepanek, P.; Borisov, O. pH-triggered reversible sol-gel transition in aqueous solutions of amphiphilic gradient copolymers. Soft Matter 2011, 7, 10824-10833.

(30) Charbonneau, C.; Lima, M. M. D. S.; Chassenieux, C.; Colombani, O.; Nicolai, T. Structure of $\mathrm{pH}$ sensitive self-assembled amphiphilic di-and triblock copolyelectrolytes: micelles, aggregates and transient networks. Physical Chemistry Chemical Physics 2013, 15, 3955-3964. 
(31) Myung, J. S.; Taslimi, F.; Winkler, R. G.; Gompper, G. Self-organized structures of attractive end-functionalized semiflexible polymer suspensions. Macromolecules 2014, 47, 4118-4125.

(32) Amin, D.; Likhtman, A. E.; Wang, Z. Dynamics in supramolecular polymer networks formed by associating telechelic chains. Macromolecules 2016, 49, 7510-7524.

(33) Tsitsilianis, C.; Serras, G.; Ko, C.-H.; Jung, F.; Papadakis, C. M.; RikkouKalourkoti, M.; Patrickios, C. S.; Schweins, R.; Chassenieux, C. Thermoresponsive Hydrogels Based on Telechelic Polyelectrolytes: From Dynamic to "Frozen" Networks. Macromolecules 2018, 51, 2169-2179.

(34) Gârlea, I. C.; Jaramillo-Cano, D.; Likos, C. N. Self-organization of gel networks formed by block copolymer stars. Soft Matter 2019, 15, 3527-3540.

(35) Williams, D. E.; Houpt, D. J. Fluorine nonbonded potential parameters derived from crystalline perfluorocarbons. Acta Crystallographica Section B 1986, 42, 286-295.

(36) Nyrkova, I. A.; Khokhlov, A. R.; Doi, M. Microdomains in block copolymers and multiplets in ionomers: parallels in behavior. Macromolecules 1993, 26, 3601-3610.

(37) Khokhlov, A. R.; Kramarenko, E. Y. Polyelectrolyte/ionomer behavior in polymer gel collapse. Macromolecular Theory and Simulations 1994, 3, 45-59.

(38) Varley, R. J.; van der Zwaag, S. Towards an understanding of thermally activated selfhealing of an ionomer system during ballistic penetration. Acta Materialia 2008, 56, $5737-5750$.

(39) Chen, Q.; Tudryn, G. J.; Colby, R. H. Ionomer dynamics and the sticky Rouse model. Journal of Rheology 2013, 57, 1441-1462.

(40) Tanaka, F.; Edwards, S. Viscoelastic properties of physically crosslinked networks. 1. Transient network theory. Macromolecules 1992, 25, 1516-1523. 
(41) Semenov, A.; Joanny, J.-F.; Khokhlov, A. Associating polymers: equilibrium and linear viscoelasticity. Macromolecules 1995, 28, 1066-1075.

(42) Rubinstein, M.; Panyukov, S. Nonaffine deformation and elasticity of polymer networks. Macromolecules 1997, 30, 8036-8044.

(43) Rubinstein, M.; Semenov, A. N. Thermoreversible gelation in solutions of associating polymers. 2. Linear dynamics. Macromolecules 1998, 31, 1386-1397.

(44) Semenov, A. N.; Rubinstein, M. Thermoreversible gelation in solutions of associative polymers. 1. Statics. Macromolecules 1998, 31, 1373-1385.

(45) Guo, L.; Luijten, E. Reversible gel formation of triblock copolymers studied by molecular dynamics simulation. Journal of Polymer Science Part B: Polymer Physics 2005, 43, 959-969.

(46) Mester, Z.; Mohan, A.; Fredrickson, G. H. Macro-and microphase separation in multifunctional supramolecular polymer networks. Macromolecules 2011, 44, 9411-9423.

(47) Sakai, T.; Matsunaga, T.; Yamamoto, Y.; Ito, C.; Yoshida, R.; Suzuki, S.; Sasaki, N.; Shibayama, M.; i. Chung, U. Design and fabrication of a high-strength hydrogel with ideally homogeneous network structure from tetrahedron-like macromonomers. Macromolecules 2008, 41, 5379.

(48) Vamvakaki, M.; Patrickios, C. S. Polyelectrolytic Amphiphilic Model Netwrks in Water: A Molecular Thermodynamic Theory for Their Microphase Separation. The Journal of Physical Chemistry B 2001, 105, 4979-4986.

(49) Georgiou, T.; Vamvakaki, M.; Patrickios, C. Microphase separation under constraints: a molecular thermodynamic theory for polyelectrolytic amphiphilic model networks in water. Polymer 2004, 45, 7341-7355. 
(50) Karbarz, M.; Stojek, Z.; Georgiou, T.; Patrickios, C. Microphase separation in ABA triblock copolymer-based model conetworks in the bulk: Effect of loop formation. Polymer 2006, 47, 5182-5186.

(51) Weeks, J. D.; Chandler, D.; Andersen, H. C. Role of Repulsive Forces in Determining the Equilibrium Structure of Simple Liquids. The Journal of Chemical Physics 1971, 54,5237 .

(52) Kremer, K.; Grest, G. S. Dynamics of entangled linear polymer melts: A moleculardynamics simulation. Journal of Chemical Physics 1990, 92, 5057-5086.

(53) Hockney, R. W.; Eastwood, J. W. Computer Simulation Using Particles; IOP: London, 1988.

(54) Deserno, M.; Holm, C. How to mesh up Ewald sums. I. A theoretical and numerical comparison of various particle mesh routines. Journal of Chemical Physics 1998, 109, 7678.

(55) Deserno, M.; Holm, C. How to mesh up Ewald sums. II. An accurate error estimate for the Particle-Particle-Particle-Mesh algorithm. Journal of Chemical Physics 1998, 109, 7694.

(56) Grest, G. S.; Kremer, K. Molecular dynamics simulation for polymers in the presence of a heat bath. Physical Review A 1986, 33, 3628-31.

(57) Weik, F.; Weeber, R.; Szuttor, K.; Breitsprecher, K.; de Graaf, J.; Kuron, M.; Landsgesell, J.; Menke, H.; Sean, D.; Holm, C. ESPResSo 4.0 - an extensible software package for simulating soft matter systems. The European Physical Journal Special Topics 2019, 227, 1789-1816.

(58) Bunker, D. L.; Hase, W. L. On non-RRKM unimolecular kinetics: Molecules in general, and CH3NC in particular. The Journal of Chemical Physics 1973, 59, 4621-4632. 
(59) Rice, O. K.; Ramsperger, H. C. Theories of unimolecular gas reactions at low pressures. Journal of the American Chemical Society 1927, 49, 1617-1629.

(60) Kassel, L. S. Studies in Homogeneous Gas Reactions. I. The Journal of Physical Chemistry 1928, 32, 225-242.

(61) Mella, M. Effect of the cluster angular momentum J and the projectile orbital momentum L on capture probability and postcollision dynamics. The Journal of Chemical Physics 2009, 131, 124309.

(62) Stevens, M. J.; Kremer, K. Form Factor of Salt-Free Linear Polyelectrolytes. Macromolecules 1993, 26, 4717.

(63) Mella, M.; Mollica, L.; Izzo, L. Influence of charged intramolecular hydrogen bonds in weak polyelectrolytes: A Monte Carlo study of flexible and extendible polymeric chains in solution and near charged spheres. Journal of Polymer Science Part B: Polymer Physics 2015, 53, 650-663.

(64) Tagliabue, A.; Izzo, L.; Mella, M. Absorbed weak polyelectrolytes: Impact of confinement, topology, and chemically specific interactions on ionization, conformation free energy, counterion condensation, and absorption equilibrium. Journal of Polymer Science Part B: Polymer Physics 2019, 57, 491-510.

(65) Tagliabue, A.; Izzo, L.; Mella, M. Impact of Charge Correlation, Chain Rigidity, and Chemical Specific Interactions on the Behavior of Weak Polyelectrolytes in Solution. The Journal of Physical Chemistry B 2019, 123, 8872-8888.

(66) Mons, P.; Schacher, F. H. work in progress. 
Graphical TOC Entry 